\title{
Nonlinear Wave Equations and Constrained Harmonic Motion
}

\author{
P. Deift ${ }^{\star}$, F. Lund ${ }^{\star \dagger}$, and E. Trubowitz ${ }^{\star \star}$ \\ Courant Institute of Mathematical Sciences, New York University, New York, NY 10012 USA \\ + University of Chile, Santiago, Chile
}

\section{Introduction}

Moser and Trubowitz [7] showed that the study of the Korteweg-de Vries equation is simply the study of constrained harmonic motion. Here we show the same is true for the nonlinear Schrödinger, sine-Gordon and Toda lattice equations. Briefly, we have found a change of variables under which these integrable wave equations become a system of free oscillators constrained to an intersection of quadrics in phase space. This is in analogy with the linear case in which the study of wave equations with constant coefficients is reduced via the Fourier transform to the study of harmonic oscillators with linear constraints. One wave equation differs from another only in the nature of the constraint and in each case the constrained system is itself integrable.

In another paper we will use the constrained particle systems to analyze the global phase space geometry of the nonlinear Schrödinger and Sine-Gordon equations. We have no doubt that our technique can be directly applied to the continuous Heisenberg spin chain, the generalized Sine-Gordon equation, the classical Thirring model and any other nonlinear wave equation associated with a second order linear problem.

\section{Hamiltonian Mechanics with Constraints ${ }^{1}$}

Let $\omega=\sum_{i=1}^{n} d x^{i} \wedge d y^{i}$ be the standard symplectic form an $R^{2 n}$ and $\{F, G\}$ the corresponding Poisson bracket between smooth functions $F$ and $G$. If $H \in C^{\infty}\left(R^{2 n}\right)$ we denote by $V_{H}$ the corresponding Hamiltonian vector field for which $d H(\cdot)=\omega\left(V_{H}, \cdot\right)$. As usual $S=\left(\begin{array}{rr}0 & I \\ -I & 0\end{array}\right)$ and $\omega(u, v)=(u, S v)$.

* Research supported by National Science Foundation Grant No. NSF-MCS-76-07039

$\star \star$ Research supported by National Science Foundation Grant No. NSF-MCS-76-18222

1 For a related discussion of mechanics with constraints see [2] 
Let $m<n$ and $\phi_{1}, \ldots, \phi_{2 m} \in C^{\infty}\left(R^{2 n}\right)$ with $d \phi_{1}, \ldots, d \phi_{2 m}$ independent on the variety $X=\left\{(x, y) \in R^{2 n}: \phi_{1}(x, y)=\phi_{2}(x, y)=\ldots=\phi_{2 m}(x, y)=0\right\} . X$ is a smoothly imbedded submanifold of $R^{2 n}$.

Lemma 1. The restriction of $\omega$ to $X, \omega_{X}$, is non-degenerate if and only if the matrix $C=\left(\left\{\phi_{i}, \phi_{j}\right\}\right)$ is non-singular at every point $p$ of $X$.

Proof. If $C$ is singular at $p$, then there are $a_{1}, \ldots, a_{2 m}$, not all zero, such that $\left(\nabla \phi_{j}, S\left(\sum_{i=1}^{2 m} a_{i} \nabla \phi_{i}\right)\right)=\sum_{i=1}^{2 m} a_{i}\left\{\phi_{j}, \phi_{i}\right\}=0$ for all $j$.

In other words $v=S\left(\sum_{i=1}^{2 m} a_{i} \nabla \phi_{i}\right) \neq 0$ is tangent to $X$ at $p$. But then $\omega(u, v)$ $=\left(u, S\left(S \sum_{i=1}^{2 m} a_{i} \nabla \phi_{i}\right)\right)=-\left(u, \sum_{i=1}^{2 m} a_{i} \nabla \phi_{i}\right)=0$ for all vectors $u \in T X_{p}$. Thus $\omega$ is degenerate on $X$.

Conversely if for some $0 \neq v \in T X_{p}, \omega(u, v)=(u, S v)=0$ for all $u \in T X_{p}$, then $S v=\sum_{i=1}^{2 m} a_{i} \nabla \phi_{i}$ for some $a_{i}$, not all zero. But then

$$
\left\{\phi_{j}, \sum_{i=1}^{2 m} a_{i} \phi_{i}\right\}=\left(\nabla \phi_{j}, S\left(\sum_{i=1}^{2 m} a_{i} \nabla \phi_{i}\right)\right)=0 \quad \text { as } S\left(\sum_{i=1}^{2 m} a_{i} \nabla \phi_{i}\right)=-v \in T X_{p}
$$

The form $\omega_{X}$ is closed since $d\left(\omega_{X}\right)=(d \omega)_{X}=0$ and therefore, if $C$ is non-singular, $\omega_{X}$ defines a symplectic structure on $X$. From now on we will always assume that (the antisymmetric matrix) $C^{-1}=\left(c_{i j}^{-1}\right)$ exists. Also, if $H \in C^{\infty}\left(R^{2 n}\right), V_{H}^{\prime}$ will denote the Hamiltonian vector field on $X$ for $\left.H\right|_{X}$ with respect to $\omega_{X}$.

In general $V_{H}^{\prime} \neq V_{H}$ on $X$. We have

Lemma 2. $V_{H}^{\prime}=V_{H}$ on $X$ if and only if $\left\{H, \phi_{j}\right\}=0$ on $X$ for all $j=1, \ldots, 2 m$. Furthermore, if $H_{0} \in C^{\infty}\left(R^{2 n}\right)$ and $\alpha_{i}=\sum_{i=1}^{2 m} c_{i j}^{-1}\left\{H_{0}, \phi_{j}\right\}, i=1, \ldots, 2 m$, then $V_{H_{0}}^{\prime}=V_{H}$ on $X$ where $H=H_{0}+\sum_{i=1}^{2 m} \alpha_{i} \phi_{i}$.

Proof. First of all if $\left\{H, \phi_{j}\right\}=\left(S \nabla H, \nabla \phi_{j}\right)=0$ on $X$ for $j=1, \ldots, 2 m$, then $V_{H}=S \nabla H \in T X_{p}$ for all $p \in X$ so that $V_{H}^{\prime}=V_{H}$. Conversely if $S \nabla H=V_{H_{0}}^{\prime}=V_{H}^{\prime} \in T X_{p}$ for all $p \in X$, then $\left\{H, \phi_{i}\right\}=\left(S \nabla H, \nabla \phi_{i}\right)=0$ for all $i=1, \ldots, 2 m$. on $X$.

The second part of the lemma follows from the first by noting that $V_{H_{0}}^{\prime}=V_{H}^{\prime}=V_{H}$

All Poisson brackets on $X$ can be calculated in the ambient space $R^{2 n}$ by the following

Lemma 3. Let $\{F, G\}_{X}$ be the Poisson bracket for $\omega_{X}$. Then $\{F, G\}_{X}=\{F, G\}$ $-\sum_{i, j=1}^{2 m}\left\{F, \phi_{i}\right\} c_{i j}^{-1}\left\{\phi_{j}, G\right\}$ on $X$ where the right hand side is calculated for any smooth extensions of $F$ and $G$ to an open neighborhood of $X$ in $R^{2 n}$. 
Proof. Suppose that $\left\{F, \phi_{i}\right\}=\left\{G, \phi_{i}\right\}=0$ on $X$ for all $i$. Then by Lemma 2, $\{F, G\}_{X}=\omega_{X}\left\{V_{F}^{\prime}, V_{G}^{\prime}\right\}=\omega\left(V_{F}, V_{G}\right)=\{F, G\}$. For general $F$ and $G$, again by Lemma 2,

$$
\begin{aligned}
\{F, G\}_{X}= & \left\{F+\sum_{i, j=1}^{2 m} c_{i j}^{-1}\left\{F, \phi_{j}\right\} \phi_{i}, G+\sum_{k, l=1}^{2 m} c_{k l}^{-1}\left\{G, \phi_{l}\right\} \phi_{k}\right\}_{X} \\
= & \left\{F+\sum_{i, j=1}^{2 m} c_{i j}^{-1}\left\{F, \phi_{j}\right\} \phi_{i}, G+\sum_{k, l=1}^{2 m} c_{k l}^{-1}\left\{G, \phi_{l}\right\} \phi_{k}\right\} \\
= & \{F, G\}+\sum_{k, l=1}^{2 m} c_{k l}^{-1}\left\{G, \phi_{l}\right\}\left\{F, \phi_{k}\right\} \\
& +\sum_{i, j=1}^{2 m} c_{i j}^{-1}\left\{F, \phi_{j}\right\}\left\{\phi_{i}, G\right\} \\
& \left.+\sum_{i, j, k, l=1}^{2 m} c_{i j}^{-1} c_{k l}^{-1}\left\{F, \phi_{j}\right\}\left\{G, \phi_{l}\right\} c_{i k} \quad \text { (on } X\right) \\
= & \{F, G\}-\sum_{i, j=1}^{2 m}\left\{F, \phi_{i}\right\} c_{i j}^{-1}\left\{\phi_{j}, G\right\} .
\end{aligned}
$$

This establishes the formula.

The significance of the above lemmas is made clear in the following example of constrained harmonic motion.

Let

$$
\begin{aligned}
& H_{0}=\frac{1}{2} \sum_{i=1}^{n} y_{i}^{2}+\frac{1}{2} \sum_{i=1}^{n} \sigma_{i} x_{i}^{2} . \\
& \phi_{1}=\sum_{i=1}^{n} x_{i}^{2}-1, \quad \phi_{2}=\sum_{i=1}^{n} x_{i} y_{i} .
\end{aligned}
$$

$X=\left\{\phi_{1}=\phi_{2}=0\right\}$ is the tangent bundle to the unit sphere.

We have $C_{12}=\left\{\phi_{1}, \phi_{2}\right\}=2\left(\phi_{1}+1\right),\left\{\phi_{1}, H_{0}\right\}=2 \phi_{2},\left\{\phi_{2}, H_{0}\right\}=\sum_{i=1}^{n} y_{i}^{2}$ $-\sum_{i=1}^{n} \sigma_{i} x_{i}^{2}$ and $H=H_{0}+\alpha_{1} \phi_{1}+\alpha_{2} \phi_{2}$ where $\alpha_{1}, \alpha_{2}$ are determined by

$$
\begin{aligned}
0=\left\{\phi_{1}, H\right\} & =2 \phi_{2}+\alpha_{2} 2\left(\phi_{1}+1\right)=2 \alpha_{2}, \\
0=\left\{\phi_{2}, H\right\} & =\sum_{i=1}^{n} y_{i}^{2}-\sum_{i=1}^{n} \sigma_{i} x_{i}^{2}-\alpha_{1}\left[2\left(\phi_{1}+1\right)\right] \\
& =\sum_{i=1}^{n} y_{i}^{2}-\sum_{i=1}^{n} \sigma_{i} x_{i}^{2}-2 \alpha_{1} .
\end{aligned}
$$

on $X$, so that

$$
\alpha_{1}=\frac{1}{2} \sum_{i=1}^{n} \mathrm{y}_{i}^{2}-\frac{1}{2} \sum_{i=1}^{n} \sigma_{i} \mathrm{x}_{i}^{2}
$$

and

$\alpha_{2}=0$. 
Thus

$$
\begin{aligned}
H= & \frac{1}{2} \sum_{i=1}^{n} y_{i}^{2}+\frac{1}{2} \sum_{i=1}^{n} \sigma_{i} x_{i}^{2} \\
& +\left(\frac{1}{2} \sum_{i=1}^{n} y_{i}^{2}-\frac{1}{2} \sum_{i=1}^{n} \sigma_{i} x_{i}^{2}\right)\left(\sum_{i=1}^{n} x_{i}^{2}-1\right) .
\end{aligned}
$$

Now the Hamiltonian equations of motion for the system of the harmonic oscillators forced to remain on the unit sphere are:

$$
\begin{aligned}
\frac{d}{d t} x_{i} & =\left\{x_{i}, H_{0}\right\}_{X}=\left\{x_{i}, H\right\}=y_{i}, \\
\frac{d}{d t} y_{i} & =\left\{y_{i}, H_{0}\right\}_{X}=\left\{y_{i}, H\right\} \\
& =-\sigma_{i} x_{i}+\left(\frac{1}{2} \sum_{j=1}^{n} y_{j}^{2}-\frac{1}{2} \sum_{j=1}^{n} \sigma_{j} x_{j}^{2}\right)\left(-2 x_{i}\right),
\end{aligned}
$$

1.e.,

$$
\frac{d^{2}}{d t^{2}} x_{i}+\sigma_{i} x_{i}=\left(\sum_{j=1}^{n}\left(\sigma_{j} x_{j}^{2}-y_{j}^{2}\right)\right) x_{i} .
$$

The vector

$$
\left(\sum_{i=1}^{n}\left(\sigma_{i} x_{i}^{2}-y_{i}^{2}\right)\right)\left(\begin{array}{c}
x_{1} \\
\vdots \\
x_{n}
\end{array}\right)=\left(-2 \alpha_{1}\right)\left(\begin{array}{c}
x_{1} \\
\vdots \\
x_{n}
\end{array}\right)
$$

is the (normal) force required to constrain the oscillators.

In this example

$$
\begin{aligned}
\{F, G\}_{X} & =\{F, G\}-\left(\left\{F, \phi_{1}\right\},\left\{F, \phi_{2}\right\}\right)\left(\begin{array}{cc}
0 & -\frac{1}{2} \\
\frac{1}{2} & 0
\end{array}\right)\left(\begin{array}{l}
\left\{\phi_{1}, G\right\} \\
\left\{\phi_{2}, G\right\}
\end{array}\right) \\
& =\{F, G\}+\frac{1}{2}\left[\left\{F, \phi_{1}\right\}\left\{\phi_{2}, G\right\}-\left\{F, \phi_{2}\right\}\left\{\phi_{1}, G\right\}\right] .
\end{aligned}
$$

This system was first discussed by Neumann (1859) in the case $n=3$. Remarkably, the motion is integrable by:

Theorem 1. The functions

$$
A_{j}=x_{j}^{2}+\sum_{i \neq j} \frac{\left(x_{i} y_{j}-x_{j} y_{i}\right)^{2}}{a_{j}-a_{i}}, \quad j=1, \ldots, n,
$$

Poisson commute on $R^{2 n}$ and on $X$. Moreover

$$
\begin{aligned}
\sum_{j=1}^{n} A_{j} & =\phi_{1}+1 \\
& =1 \text { on } X,
\end{aligned}
$$

and

$$
\begin{aligned}
\sum_{j=1}^{n} a_{j} A_{j} & =\sum_{j=1}^{n} a_{j} x_{j}^{2}+\left(\sum_{j=1}^{n} x_{j}^{2}\right)\left(\sum_{j=1}^{n} y_{j}^{2}\right)-\left(\sum_{j=1}^{n} x_{j} y_{j}\right)^{2} \\
& =2 H_{0} \text { on } X .
\end{aligned}
$$


Proof. Let $z_{i j}=x_{i} y_{j}-x_{j} y_{i}, d_{i j}=\frac{1}{a_{i}-a_{j}}$. For $j \neq k$,

$$
\begin{aligned}
\left\{A_{j}, A_{k}\right\}= & \left\{x_{j}^{2}, \sum_{l \neq k} d_{k l} z_{k l}^{2}\right\}+\left\{\sum_{i \neq j} d_{j i} z_{i j}^{2}, x_{k}^{2}\right\} \\
& +\left\{\sum_{i \neq j} d_{j i} z_{i j}^{2}, \sum_{l \neq k} d_{k l} z_{k l}^{2}\right\} \\
= & -4 x_{j} x_{k} z_{j k} d_{k j}+4 z_{k j} x_{k} x_{j} d_{j k} \\
& +\sum_{\substack{i \neq j, k \\
l=k}} d_{j i} d_{k l}\left\{z_{i j}^{2}, z_{l k}^{2}\right\}+\sum_{l \neq k} d_{j k} d_{k l}\left\{z_{k j}^{2}, z_{l k}^{2}\right\} \\
= & \sum_{i \neq j, k} d_{j i} d_{k i} 4 z_{i j} z_{i k} z_{j k} \\
& +\sum_{i \neq j, k} d_{j i} d_{k j} 4 z_{i j} z_{j k} z_{k i} \\
& +\sum_{l \neq k, j} d_{j k} d_{k l} 4 z_{k j} z_{k l} z_{j l} \\
= & 4 \sum_{i \neq j, k} z_{i j} z_{i k} z_{j k}\left[d_{j i} d_{k i}+d_{i j} d_{k j}+d_{j k} d_{i k}\right] \\
= & 0
\end{aligned}
$$

as

$$
d_{j i} d_{k i}+d_{i j} d_{k j}+d_{j k} d_{i k}=0 .
$$

It is enough to prove this when $a_{i}$, say, is zero. But then

$$
\begin{aligned}
& d_{j i} d_{k i}+d_{i j} d_{k j}+d_{j k} d_{i k} \\
& \quad=\frac{1}{a_{j}} \frac{1}{a_{k}}+\frac{1}{\left(-a_{j}\right)} \frac{1}{a_{k}-a_{j}}+\frac{1}{a_{j}-a_{k}} \frac{1}{\left(-a_{k}\right)} \\
& \quad=\frac{1}{a_{j} a_{k}}+\frac{1}{a_{j} a_{k}\left(a_{k}-a_{j}\right)}\left[-a_{k}+a_{j}\right] \\
& \quad=0 .
\end{aligned}
$$

The identity $\phi_{1}+1=\sum_{j=1} \mathrm{~A}_{j}$ follows from the antisymmetry of $z_{i j}^{2} c_{i j}$ and

$$
\begin{aligned}
\sum_{j=1}^{n} a_{j} A_{j}= & \sum_{j} a_{j} x_{j}^{2}+\sum_{j} \sum_{i \neq j} \frac{a_{j}}{a_{j}-a_{i}}\left(x_{i} y_{j}-x_{j} y_{i}\right)^{2} \\
= & \sum_{j} a_{j} x_{j}^{2}+\frac{1}{2}\left[\sum_{j} \sum_{i \neq j} \frac{a_{j}}{a_{j}-a_{i}}\left(x_{i} y_{j}-x_{j} y_{i}\right)^{2}\right. \\
& \left.+\sum_{i} \sum_{i \neq j} \frac{a_{j}}{a_{i}-a_{j}}\left(x_{j} y_{i}-x_{i} y_{j}\right)^{2}\right] \\
= & \sum_{j} a_{i} x_{j}^{2}+\frac{1}{2}\left[\sum_{j} \sum_{i \neq j} \frac{a_{j}-a_{i}}{a_{j}-a_{i}}\left(x_{i} y_{j}-x_{j} y_{i}\right)^{2}\right] \\
= & \sum_{i} a_{i} x_{j}^{2}+\frac{1}{2}\left[\sum_{i, j} x_{i}^{2} y_{j}^{2}+x_{j}^{2} y_{i}^{2}-2 x_{i} y_{i} x_{j} y_{j}\right] \\
= & \sum_{j} a_{j} x_{j}^{2}+\left(\sum_{j} x_{j}^{2}\right)\left(\sum_{j} y_{j}^{2}\right)-\left(\sum_{j} x_{j} y_{j}\right)^{2} .
\end{aligned}
$$


Clearly $\left\{\phi_{1}, A_{j}\right\}=0, j=1, \ldots, n$, so that

$$
\begin{aligned}
\left\{A_{j}, A_{k}\right\}_{X} & =\left\{A_{j}, A_{k}\right\}+\frac{1}{2}\left(\left\{A_{j}, \phi_{1}\right\}\left\{\phi_{2}, A_{k}\right\}-\left\{A_{j}, \phi_{2}\right\}\left\{\phi_{1}, A_{k}\right\}\right) \\
& =0
\end{aligned}
$$

Furthermore on $X, \sum_{j=1} a_{j} A_{j}=\sum_{j=1} a_{j} x_{j}^{2}+\sum_{j=1} y_{j}^{2}=2 H_{0}$, and the proof is finished.

\section{The Korteweg de Vries Equation}

Here we will show how the solution of the $\mathrm{KdV}$ equation on the line reduces to the study of a continuum of constrained harmonic oscillators. This is in contrast to the periodic case of Moser and Trubowitz which involves a countable number of oscillators.

The results of the previous section are finite dimensional but provide a framework for the infinite dimensional problem. We will verify the facts we need directly.

Let $q(x) \in \mathscr{S}(\mathbb{R})$ be real and let $f_{k}(x)_{x \rightarrow+\infty}^{\sim} e^{i k x}, g_{k}(x)_{x \rightarrow-\infty}^{\sim} e^{-i k x}$ be the Jost solutions $^{2}$ for the Schrödinger operator $-d^{2} / d x^{2}+q(x)$. The solutions $f_{k}(x), g_{k}(x)$ are analytic in the upper half $k$-plane and related on the real axis by the reflection and transmission coefficients $R(k)=R(k ; q) \in \mathscr{S}, T(k)=T(k ; q)$ through

$$
T(k) g_{k}(x)=R(k) f_{k}(x)+f_{-k}(x) .
$$

For simplicity we will always assume that $-d^{2} / d x^{2}+q(x)$ has no bound states or resonances so that $T(k)$ is analytic in the upper half plane and $T(0)=0=1+R(0)$.

We begin by deriving an identity among the functions $f_{k}^{2},-\infty<k<\infty$, from the estimate ${ }^{3}$

$$
T(k) f_{k}(x) g_{k}(x)=1+0\left(\frac{1}{k^{2}}\right), \quad \operatorname{Im} k \geqq 0 .
$$

Multiplying by $i / \pi k$ and integrating clockwise along a semicircle $C_{r}$ of radius $r$, we obtain

$$
\begin{aligned}
& \frac{i}{\pi} \int_{C_{r}} \frac{d k}{k}+0\left(\frac{1}{r^{2}}\right)=\frac{i}{\pi} \int_{C_{r}} \frac{T(k) f_{k}(x) g_{k}(x)}{k} d k \\
& =\frac{i}{\pi} \int_{|k|>\varepsilon} \frac{T(k) f_{k}(x) g_{k}(x)}{k} d k+\frac{i}{\pi} \int_{C_{\varepsilon}} \frac{T(k) f_{k}(x) g_{k}(x)}{k} d k \\
& =\frac{i}{\pi} \int_{|k|>\varepsilon} \frac{R(k) f_{k}^{2}(x)}{k} d k+\frac{i}{\pi} \int_{C_{\varepsilon}} \frac{T(k) f_{k}(x) g_{k}(x) d k}{k} .
\end{aligned}
$$

2 See Deift and Trubowitz [3a], Sects. 1 and 2, for the necessary information on scattering theory

3 See Deift and Trubowitz [3a], Sect. 4 
In the second line Cauchy's theorem is used and in the third the fact that $f_{k}(x) f_{-k}(x) / k$ is odd. Letting $r \rightarrow \infty$ we find

$$
1=\frac{i}{\pi} \int_{|k|>\varepsilon} \frac{R(k) f_{k}^{2}(x)}{k} d k+\frac{i}{\pi} \int_{C_{\varepsilon}} \frac{T(k) f_{k}(x) g_{k}(x)}{k} d k,
$$

then letting $\varepsilon \downarrow 0$

$$
1=\frac{i}{\pi} \int_{-\infty}^{\infty} \frac{R(k) f_{k}^{2}(x)}{k} d k
$$

where the integral is interpreted as a principal value.

Now differentiate (1) twice to obtain

$$
q(x)=\frac{i}{\pi} \int_{-\infty}^{\infty} k R(k) f_{k}^{2}(x) d k-\frac{i}{\pi} \int_{-\infty}^{\infty} \frac{R(k)}{k} f_{k}^{\prime 2}(x) d x .
$$

Here the differential equation for $f_{k}$ is used and the principal value is easily handled.

Also

$$
\frac{d}{d x}\left\{\frac{i}{\pi} \int_{-\infty}^{\infty} k R(k) f_{k}^{2}(x) d k+\frac{i}{\pi} \int_{-\infty}^{\infty} \frac{R(k)}{k} f_{k}^{\prime 2}(x) d k\right\}=0
$$

so that

$$
q(x)=\frac{2 i}{\pi} \int_{-\infty}^{\infty} k R(k) f_{k}^{2}(x) d k
$$

where the constant of integration vanishes by Riemann-Lebesgue. It is also possible to derive (3) directly by contour integration (see [3, Sect. 4]).

Fix a real $p(x)$ in $\mathscr{S}(\mathbb{R})$ and set

$$
M=\{\text { real } q \in \mathscr{S}:|R(k ; q)|=|R(k ; p)|,-\infty<k<\infty\} .
$$

$M$ is closed in the topology it inherits from $\mathscr{S}$ because $R(k ; q)$ is a continuous function of $q$ for each $k \neq 0^{4}$ and continuous in $k$ for each $q$.

Lemma $1 .^{5}$ If $R(k ; p)$ vanishes to infinite order at some $k \neq 0, M$ is not simply connected.

Proof. For clarity and simplicity we make the proof when $R(k ; p) \neq 0$ for $-1<k<1, \quad|k|>2$ and $R(k ; p)$ vanishes for $|k|$ between 1 and 2 . Set $R_{s}(k) \rightarrow=e^{2 \pi i s n(k)} R(k ; p), 0 \leqq s \leqq 1$, where

$$
\begin{array}{rlrlrl}
n(k) & =0 & \text { for } & & -1<k<1 \\
& =1 \text { for } & k>2 \\
& =-1 \text { for } & k<-2 .
\end{array}
$$

$4 R(0 ; q)$ is continuous on $M$ but not in $\mathscr{S}$

5 For more information on the phase space geometry of the KdV equation, see Deift and Trubowitz [3b] 
By the basic result of inverse scattering theory there is a continuous, closed curve $q_{s} \in M, 0 \leqq s \leqq 1$, with $R\left(k ; q_{s}\right)=R_{s}(k)$ and $q_{0}=q_{1}=p$. We will show that this curve cannot be contracted to $p$. Suppose it is contractible with homotopy $q_{s}(x ; u) \in M$, $0 \leqq u \leqq 1$. The corresponding reflection coefficients $R_{s}(k ; u)=R\left(k ; q_{s}(x, u)\right)$ are jointly continuous in $s, u$, and $k$ and never vanish for $|k| \notin[1,2]$ so that $O(k ; s, u)$ $=\operatorname{Im}\left(\log R_{s}(k ; u)\right)$ can be chosen as a continuous function in $\{|k|>2\} \times[0,1] \times[0,1]$ and $\{-1<k<1\} \times[0,1] \times[0,1]$ with $\theta(0 ; s, u)=\pi$. Similarly we can choose $\theta(k ; p)=\operatorname{Im}(\log R(k ; p))$ continuous on $\{|k|>2\}$ and $\{-1<k<1\}$ with $\theta(0 ; p)=\pi$.

Now for $|k|>2, \theta(k ; s, u)$ must satisfy

$$
\begin{array}{llrl}
\theta(k ; 0, u) & =\theta(k ; p)(\bmod 2 \pi) & & 0 \leqq u \leqq 1, \\
\theta(k ; 1, u) & =\theta(k ; p)(\bmod 2 \pi), & & 0 \leqq u \leqq 1, \\
\theta(k ; s, 0)=\theta(k ; p)(\bmod 2 \pi), & & 0 \leqq s \leqq 1, \\
\theta(k ; s, 1)=2 \pi s+\theta(k ; p)(\bmod 2 \pi), & & 0 \leqq s \leqq 1 .
\end{array}
$$

But one easily checks that this is inconsistent with the continuity of $\theta(k ; s, u)$. Hence $M$ is not simply connected.

On the other hand, if $|R(k ; p)|>0$ for all $k$, then $M$ is simply connected. To see this define

$$
\theta(k ; q)=\operatorname{Im}(\log R(k ; q))
$$

as a continuous function of $-\infty<k<\infty$ for each fixed $q \in M$. Because $R(0, a)=-1, \theta(0 ; q)=\pi$ and it follows easily that $\theta(k ; q)$ is jointly continuous in $-\infty<k<\infty$ and $q \in M$. An elementary argument now shows that $M$ is simply connected; indeed for any continuous, closed curve $q_{s} \in M, 0 \leqq s \leqq 1$ with corresponding reflection coefficients $R_{s}(k)=|R(k ; p)| e^{i \theta\left(k ; q_{s}\right)}$, the required homotopy is $R_{s}(k ; u)=|R(k ; p)| e^{i\left[u \theta\left(k ; q_{s}\right)+(1-u) \theta\left(k ; q_{0}\right)\right]}, 0 \leqq s, u \leqq 1$. For the remainder of this section we will assume that $r_{k}=r_{-k} \equiv|R(k ; p)|>0,-\infty<k<\infty$.

Before we can continue it is necessary to make a lengthy technical digression.

Define the Hilbert spaces $\mathscr{H}_{2} \subset \mathscr{H}_{1}$ of functions $z_{l}=\left(x_{l}, y_{l}\right),-\infty<l<\infty$, as the closures of $\mathscr{C}_{0}^{\infty}(\mathbb{R}) \times \mathscr{C}_{0}^{\infty}(\mathbb{R})$ with respect to the norms

$$
\|z\|_{1}^{2}=\left|z_{0}\right|^{2}+\int_{-\infty}^{\infty} \frac{\left|z_{l}-z_{0}\right|^{2}}{l^{2}\left(1+l^{2}\right)} d l
$$

and

$$
\begin{aligned}
\|z\|_{2}^{2}= & \left|z_{0}\right|^{2}+\left|\left(\frac{d z}{d l}\right)_{0}\right|^{2}+\int_{-\infty}^{\infty} \frac{\left|z_{l}-z_{0}\right|^{2}}{l^{2}\left(1+l^{2}\right)^{4} r_{l}^{2}} d l \\
& +\int_{-\infty}^{\infty} \frac{\left|\frac{z_{l}-z_{0}}{l}-\left(\frac{d z}{d l}\right)\right|_{0}^{2}}{l^{2}\left(1+l^{2}\right)} d l .
\end{aligned}
$$

Functions in $\mathscr{H}_{2}$ have one $L^{2}$-derivative at zero whereas functions in $\mathscr{H}_{1}$ are merely continuous. 
If $L\left(w_{1}\right)$ is a bounded linear functional on $\mathscr{H}_{1}$, then by Reisz $L\left(w_{1}\right)=\left(w_{L}, w_{1}\right)^{6}$ with $w_{L} \in \mathscr{H}_{1}$.

By definition

a bounded linear functional $L\left(w_{1}\right)$ has property 1 if $w_{L} \in \mathscr{H}_{2}$;

a bounded bilinear form $B\left(w_{1}, w_{2}\right)$ has property 2 if $B\left(\cdot, w_{2}\right)$, and $B\left(w_{1}, \cdot\right)$ have property 1 for all $w_{1}, w_{2}$;

a bounded trilinear form $T\left(w_{1}, w_{2}, w_{3}\right)$ has property 3 if $T\left(\cdot, \cdot, w_{3}\right), T\left(\cdot, w_{2}, \cdot\right)$ and $T\left(w_{1}, \cdot, \cdot\right)$ have property 2 for all $w_{1}, w_{2}, w_{3}$, and so on.

We now introduce an algebra $\mathscr{A}$ of complex valued functions on $\mathscr{H}_{1}$ for which a suitable Poisson bracket can be defined. A function $F(z)$ is in $\mathscr{A}$ if

(i) $F(z)$ has Frechet derivatives on $\mathscr{H}_{1}$ of all orders.

$$
\begin{aligned}
& F^{\prime}(z)(w)=(D F(z), w), \quad D F(z) \in \mathscr{H}_{1} \\
& F^{\prime \prime}(z)\left(w_{1}, w_{2}\right)=\left(D^{2} F(z) w_{1}, w_{2}\right),
\end{aligned}
$$

$D^{2} F(z)$ is bounded from $\mathscr{H}_{1}$ to $\mathscr{H}_{1}$

$F^{\prime \prime \prime}(z)\left(w_{1}, w_{2}, w_{3}\right)=\left(D^{3} F(z)\left(w_{1}\right) w_{2}, w_{3}\right)$

$w_{1} \rightarrow D^{3} F(z)\left(w_{1}\right)$ maps $\mathscr{H}_{1}$ continuously into the bounded maps from $\mathscr{H}_{1}$ to $\mathscr{H}_{1}$

and so on.

(ii) For all $z, w_{1}, w_{2}, \ldots$ in $\mathscr{H}_{1}^{7}$

$$
F^{\prime}\left(w_{1}\right)=\lim _{n} F^{\prime}(z)\left(\chi_{n} w_{1}\right)
$$

$$
F^{\prime \prime}(z)\left(w_{1}, w_{2}\right)=\lim _{n} F^{\prime \prime}(z)\left(\chi_{n} w_{1}, w_{2}\right)
$$

$F^{\prime \prime \prime}(z)\left(w_{1}, w_{2}, w_{3}\right)=\lim _{n} F^{\prime \prime \prime}(z)\left(\chi_{n} w_{1}, w_{2}, w_{3}\right)$

etc., where $\chi_{n}(l)$ is one on $\left\{|l| \geqq \frac{1}{n}\right\}$ and zero otherwise.

(iii) $F^{(n)}(z)\left(w_{1}, \ldots, w_{n}\right)$ has property $n$ for each $n \geqq 1$, continuously in $z$, i.e.,

$z \mapsto D F(z) \quad$ maps $\mathscr{H}_{1}$ continuously into $\mathscr{H}_{2}$

$z \mapsto D^{2} F(z) w \quad$ maps $\mathscr{H}_{2}$ continuously into $\mathscr{H}_{2}$ for each $w$.

It is easy to check that $\mathscr{A}$ is an algebra.

If $F \in \mathscr{A}$, then by (iii), $D^{2} F(z)$ maps $\mathscr{H}_{1}$ into $\mathscr{H}_{2}$ for each $z$. This map is necessarily continuous: indeed, by the closed graph theorem it suffices to show $w_{n} \rightarrow w$ in $\mathscr{H}_{1}, D^{2} F(z) w_{n} \rightarrow z_{1}$ in $\mathscr{H}_{2}$, implies $D^{2} F(z) w=z_{1}$. But this follows from the inequality $\left\|D^{2} F(z) w_{n}-z_{1}\right\|_{1} \leqq$ const $\left\|D^{2} F(z) w_{n}-z_{1}\right\|_{2}$ and the boundedness of $D^{2} F(z)$ from $\mathscr{H}_{1}$ to $\mathscr{H}_{1}$. Moreover $\left\|D^{2} F(z)\right\|_{\mathscr{H}_{1} \rightarrow \mathscr{H}_{2}}$ is locally bounded by the uniform boundedness principle and the continuity of $z \mapsto D^{2} F(z) w$ for each $w$. Similar considerations apply to the higher derivatives.

6 We reserve the symbol $(\cdot, \cdot)$ for a real inner product so that $\|z\|_{1}^{2}=(\bar{z}, z)$, etc.

7 Note that the existence of $\lim _{n} F^{\prime}(z)\left(\chi_{n} w\right)$, say, does not follow from the boundedness of $F^{\prime}(z)$ as $\chi_{n} w$ converges to $w$ in $\mathscr{H}_{1}$ if and only if $w_{0}=0$ 
Lemma 2. The functions

$$
\phi_{1}(z)=\left(\int \frac{r_{l} x_{l}^{2}}{l} d l\right)-1, \quad \phi_{2}(z)=\int \frac{r_{l} x_{l} y_{l}}{l} d l
$$

and

$$
H_{0}(z)=\frac{1}{2} \int r_{l}\left(l x_{l}^{2}+\frac{y_{l}^{2}}{l}\right) d l
$$

defined as principal values, are in $\mathscr{A}$.

Proof. We only consider $\phi_{1}(z)$. The proofs for $\phi_{2}(z)$ and $H_{0}(z)$ are similar.

First,

$$
\begin{aligned}
\phi_{1}(z)+1 & =\lim _{n \rightarrow \infty} \int_{|l|>1 / n} \frac{r_{l} x_{l}^{2}}{l} d l \\
& =\lim _{n \rightarrow \infty}\left[\int_{1 / n<|l|<1} \frac{r_{l} x_{l}^{2}}{l} d l+\int_{|l| \geqq 1} \frac{r_{l} x_{l}^{2}}{l} d l\right] \\
& =\lim _{n \rightarrow \infty}\left[\int_{1 / n<|l|<1} \frac{r_{l}}{l}\left(x_{l}^{2}-x_{0}^{2}\right) d l+\int_{|l| \geqq 1} \frac{r_{l} x_{l}^{2}}{l} d l\right]
\end{aligned}
$$

and elementary estimates show that $\phi_{1}(z)$ is defined for all $z \in \mathscr{H}_{1}$ with $\left|\phi_{1}(z)\right| \leqq$ const $\|z\|_{1}^{2}$. Suppose that $z_{l}=\left(x_{l}, y_{l}\right), w=\left(u_{l}, v_{l}\right) \in \mathscr{H}_{1}$. Then

$$
\phi_{1}(z+w)=\phi_{1}(z)+\left[2 \int \frac{r_{l} r_{l} u_{l}}{l} d l\right]+\frac{1}{2}\left[2 \int \frac{r_{l} u_{l}^{2}}{l} d l\right] .
$$

Similar estimates to those above show that

$$
\left|\int \frac{r_{l} y_{l} u_{l}}{l} d l\right| \leqq \text { const }\|z\|_{1}\|w\|_{1}
$$

and

$$
\left|\int \frac{r_{l} u_{1, l} u_{2, l}}{l} d l\right| \leqq \text { const }\left\|w_{1}\right\|\left\|w_{2}\right\| .
$$

It follows that $\phi_{1}(z)$ is differentiable with $\phi_{1}^{(n)}(z)=0$ for $n \geqq 3$. This verifies (i) and condition (ii) is immediate.

We now calculate $D \phi_{1}(z)$ from the formula

$$
2 \int \frac{r_{l} x_{l} u_{l}}{l}=\left(\left(D \phi_{1}\right)_{0}, w_{0}\right)+\int \frac{\left(\left(D \phi_{1}\right)_{l}-\left(D \phi_{1}\right)_{0}, w_{l}-w_{0}\right)}{l^{2}\left(1+l^{2}\right)} d l
$$

for all $w=\left(u_{l}, v_{l}\right) \in \mathscr{H}_{1}$. Setting $u_{l}=0$ we find that the second component of $\left(D \phi_{1}\right)_{l}$ is zero. Setting $u_{0}=0$ and then $u_{l}=u_{0}$ we find

$$
\left(D \phi_{1}\right)_{l}-\left(D \phi_{1}\right)_{0}=\left(2 l\left(1+l^{2}\right) r_{l} x_{l}, 0\right)
$$

and

$$
\left(D \phi_{1}\right)_{0}=\left(2 \int \frac{r_{l} x_{l}}{l} d l, 0\right)
$$


Thus

$$
\left(D \phi_{1}(z)\right)_{l}=\left(\left(2 \int \frac{r_{k} x_{k}}{k} d k\right)+2 l\left(1+l^{2}\right) r_{l} x_{l}, 0\right) .
$$

A similar calculation shows that

$$
F^{\prime \prime}(z)\left(w_{1}, w_{2}\right)=\left(D^{2} \phi_{1}(z) w_{1}, w_{2}\right)
$$

where $D^{2} \phi_{1}(z)$ is the real symmetric bounded linear operator

$$
D^{2} \phi_{1}(z) w=\left(\left[2 \int \frac{r_{k} u_{k}}{k} d k\right]+2 l\left(1+l^{2}\right) r_{l} u_{l}, 0\right) .
$$

From these formulae it is clear that $z \rightarrow D \phi_{1}(z)$ maps $\mathscr{H}_{1}$ continuously to $\mathscr{H}_{2}$ and that $D^{2} \phi_{1}$ maps $\mathscr{H}_{1}$ to $\mathscr{H}_{2}$ independently of $z$. This proves (iii) and the lemma.

Lemma 3. $\mathscr{A}$ is closed under the Poisson bracket

$$
\{F, G\}(z) \equiv F^{\prime}(z)(S \delta(D G(z))),
$$

where

$$
(\delta w)_{l}=\frac{w_{l}-w_{0}}{l}
$$

and

$$
(S w)_{l}=S_{l} w_{l}=\left(\begin{array}{cc}
0 & \frac{1}{r_{l}\left(1+l^{2}\right)} \\
-\frac{1}{r_{l}\left(1+l^{2}\right)} & 0
\end{array}\right)\left(\begin{array}{l}
u_{l} \\
v_{l}
\end{array}\right)
$$

Proof. We will show that $\{F, G\} \in \mathscr{A}$ for all $F, G \in \mathscr{A}$ and that $\{F, G\}$ is bilinear, skew symmetric, nondegenerate and satisfies the Jacobi identity.

The space $\mathscr{H}_{2}$ has been chosen to place $S \delta(D G(z))$ in $\mathscr{H}_{1}$, so the brackets exists. The bilinearity of $\{F, G\}$ is immediate and the skew symmetry follows from

$$
\begin{aligned}
\{F, G\}(z) & =F^{\prime}(z)(S \delta(D G(z))) \\
& =\lim _{n} F^{\prime}(z)\left(\chi_{n} S \delta(D G(z))\right) \quad \text { by (ii) } \\
& =\lim _{n} \int\left((D F)_{l}-(D F)_{0}, \chi_{n} S_{l}\left(\frac{(D G)_{l}-(D G)_{0}}{l}\right)\right) \frac{d l}{l^{2}\left(1+l^{2}\right)} \\
& =\lim _{n}-\int\left((D G)_{l}-(D G)_{0}, \chi_{n} S_{l}\left(\frac{(D F)_{l}-(D F)_{0}}{l}\right)\right) \frac{d l}{l^{2}\left(1+l^{2}\right)} \\
& =\lim _{n}-G^{\prime}(z)\left(\chi_{n} S \delta(D F(z))\right) \\
& =-G^{\prime}(z)(S \delta(D F(z))), \quad \text { again by (ii) } \\
& =-\{G, F\}(z) .
\end{aligned}
$$


In the third line we have used the fact that $\left(\chi_{n} S(\delta(D G(z)))\right)_{l=0}=0$. To see the nondegeneracy, suppose that $\{F, G\}(z)=0$ for all $G \in \mathscr{A}$. In particular this is true for $G(z)=\int\left(h_{l}, z_{l}\right) d l$ with $h_{l} \in \mathscr{C}_{0}^{\infty}(\mathbb{R} \backslash\{0\}) \times \mathscr{C}_{0}^{\infty}(\mathbb{R} \backslash\{0\})$. It is easy to show that $G$ is indeed in $\mathscr{A}$, that $(D G)_{l}=\left(\int h_{k} d k\right)+l^{2}\left(1+l^{2}\right) h_{l}$ and that $G^{(n)}(z)=0$ for $n \geqq 2$. From $\{F, G\}(z)=0$ we obtain

$$
\int\left((D F)_{l}-(D F)_{0}, S_{l} l\left(1+l^{2}\right) h_{l}\right) \frac{d l}{l^{2}\left(1+l^{2}\right)}=0
$$

and as $h_{l}$ is arbitrary in $\mathscr{C}_{0}^{\infty}(\mathbb{R} \backslash\{0\}) \times \mathscr{C}_{0}^{\infty}(\mathbb{R} \backslash\{0\})$, we conclude that $(D F)_{l}$ $-(D F)_{0}=0$. On the other hand, if we take $G(z)=\int \frac{\left(h_{l}, z_{l}\right)}{l} d l$ (principal value) with $h_{l} \in \mathscr{C}_{0}^{\infty}(\mathbb{R}) \times \mathscr{C}_{0}^{\infty}(\mathbb{R})$, then again $G$ is in $\mathscr{A},(D G)_{l}=\left[\int \frac{h_{k}}{k} d k\right]+l\left(1+l^{2}\right) h_{l}$ and $G^{(n)}(z)=0$ for $n \geqq 2$. $\{F, G\}(z)=0$ now gives

$$
\begin{aligned}
0= & \int\left((D F)_{l}-(D F)_{0},(S \delta(D G))_{l}-(S \delta(D G))_{0}\right) \frac{d l}{l^{2}\left(1+l^{2}\right)} \\
& +\left((D F)_{0},(S \delta(D G))_{0}\right) \\
= & \left((D F)_{0},(S \delta(D G))_{0}\right)
\end{aligned}
$$

but $(S \delta(D G))_{0}=\left(\begin{array}{rr}0 & 1 \\ -1 & 0\end{array}\right) h_{0}$ and $h_{0}$ is arbitrary. Thus $((D F)(z))_{l}=0$ and the form is nondegenerate.

Now we show that $\mathscr{A}$ is closed under $\{\cdot, \cdot\}$.

$$
\begin{aligned}
\{F, G\}(z+w)= & F^{\prime}(z+w)(S \delta(D G(z+w))) \\
= & F^{\prime}(z)(S \delta(D G(z+w)))+F^{\prime \prime}(z)(w, S \delta(D G(z+w))) \\
& +\int_{0}^{1}(1-t) F^{\prime \prime \prime}(z+t w)(w, w, S \delta(D G(z+w))) d t \\
= & -G^{\prime}(z+w)(S \delta(D F(z)))+F^{\prime \prime}(z)(w, S \delta(D G(z+w))) \\
& +\int_{0}^{1}(1-t) F^{\prime \prime \prime}(z+t w)(w, w, S \delta G(z+w)) \\
= & -G^{\prime}(z)(S \delta(D F(z)))-G^{\prime \prime}(z)(w, S \delta(D F(z))) \\
& +F^{\prime \prime}(z)(w, S \delta(D G(z+w))) \\
& +\int_{0}^{1}(1-t) F^{\prime \prime \prime}(z+t w)(w, w, S \delta(D G(z+w))) \\
& -\int_{0}^{1}(1-t) G^{\prime \prime \prime}(z+t w)(w, w, S \delta(D F(z))) .
\end{aligned}
$$

But

$$
F^{\prime \prime}(z)(w, S \delta(D G(z+w)))=F^{\prime \prime}(z)(w, S \delta(D G(z)))+o\left(\|w\|_{1}\right)
$$


as

$$
\begin{aligned}
& \left|F^{\prime \prime}(z)(w, S \delta(D G(z+w)))-F^{\prime \prime}(z, S \delta(D G(z)))\right| \\
& \quad \leqq \text { const }\|w\|_{1}\|S \delta(D G(z+w)-D G(z))\|_{1} \\
& \quad \leqq \text { const }\|w\|_{1}\|D G(z+w)-D G(z)\|_{2}
\end{aligned}
$$

and $z \mapsto D G(z)$ is continuous from $\mathscr{H}_{1}$ to $\mathscr{H}_{2}$. Also for $\|w\|_{1}$ bounded,

and

$$
\begin{aligned}
\left\|F^{\prime \prime \prime}(z+t w)(w, w, S \delta(D G(z+w)))\right\| & \leqq \text { const }\|w\|_{1}^{2}\|D G(z+w)\|_{2} \\
& \leqq \text { const }\|w\|_{1}^{2}
\end{aligned}
$$

$$
\left\|G^{\prime \prime \prime}(z+t w)(w, w, S \delta(D F(z)))\right\| \leqq \text { const }\|w\|_{1}^{2}\|D F(z)\|_{2} .
$$

Thus $\{F, G\}^{\prime}(z)$ exists and

$$
\begin{aligned}
\{F, G\}^{\prime}(z)(w) & =F^{\prime \prime}(z)(w, S \delta(D G(z)))-G^{\prime \prime}(z)(w, S \delta(D F(z))) \\
& =\left(w, D^{2} F(z) S \delta(D G(z))\right)-\left(w, D^{2} G(z) S \delta(D F(z))\right) .
\end{aligned}
$$

Property 2 for $\{F, G\}^{\prime}(z)$ follows immediately from the corresponding property for $F^{\prime \prime}(z)$ and $G^{\prime \prime}(z)$. Set $g(z)=S \delta(D G(z))$. As $z \mapsto D G(z)$ is continuous from $\mathscr{H}_{1}$ to $\mathscr{H}_{2}$, $g(z)$ is continuous from $\mathscr{H}_{1}$ to $\mathscr{H}_{1}$. Furthermore by the discussion preceding Lemma 2, $\left\|D^{2} F(z)\right\|_{\mathscr{H}_{1} \rightarrow \mathscr{H}_{2}}$ is locally bounded and the continuity of $D^{2} F(z) S \delta(D G(z))$ follows from the inequality

$$
\begin{aligned}
& \left\|D^{2} F(z) g(z)-D^{2} F\left(z_{0}\right) g\left(z_{0}\right)\right\|_{2} \\
& \quad \leqq\left\|D^{2} F(z)\right\|_{\mathscr{H}_{1} \rightarrow \mathscr{H}_{2}}\left\|g(z)-g\left(z_{0}\right)\right\|_{1}+\left\|\left(D^{2} F(z)-D^{2} F\left(z_{0}\right)\right) g\left(z_{0}\right)\right\|_{2} .
\end{aligned}
$$

This verifies (iii) for $\{F, G\}^{\prime}(z)$.

Higher derivatives are treated in an entirely analogous way. Thus $\mathscr{A}$ is closed under $\{F, G\}$.

Finally we verify the Jacobi identity. For $F, G, H$ in $\mathscr{A}$,

$$
\begin{aligned}
\{\{F, G\}, H\}= & \{F, G\}^{\prime}(S \delta(D H))=F^{\prime \prime}(S \delta(D H), S \delta(D G)) \\
& -G^{\prime \prime}(S \delta(D H), S \delta(D F)) \\
\{\{G, H\}, F\}= & \{G, H\}^{\prime}(S \delta(D F))=G^{\prime \prime}(S \delta(D F), S \delta(D H)) \\
& -H^{\prime \prime}(S \delta(D F), S \delta(D G)) \\
\{\{H, F\}, G\}= & \{H, F\}^{\prime}(S \delta(D G))=H^{\prime \prime}(S \delta(D G), S \delta(D F)) \\
& -F^{\prime \prime}(S \delta(D G), S \delta(D H)) .
\end{aligned}
$$

The Jacobi identity follows from the symmetry of the second derivatives. This completes the proof of the lemma.

The Poisson brackets between the functions $\phi_{1}, \phi_{2}$, and $H_{0}$ of Lemma 2 are:

$$
\begin{aligned}
\left\{\phi_{1}, \phi_{2}\right\} & =2\left(\phi_{1}+1\right) \\
\left\{\phi_{1}, H_{0}\right\} & =2 \phi_{2} \\
\left\{\phi_{2}, H_{0}\right\} & =\int\left(\frac{r_{k} y_{k}^{2}}{k}-k r_{k} x_{k}^{2}\right) d k .
\end{aligned}
$$


For example,

$$
\begin{aligned}
& D \phi_{1}=\left(2\left[\int \frac{r_{k} x_{k}}{k} d k\right]+2 l\left(1+l^{2}\right) r_{l} x_{l}, 0\right) \\
& D \phi_{2}=\left(\left[\int \frac{r_{k} y_{k}}{k} d k\right]+l\left(1+l^{2}\right) r_{l} y_{l},\left[\int \frac{r_{k} x_{k}}{k} d k\right]+l\left(1+l^{2}\right) r_{l} x_{l}\right)
\end{aligned}
$$

so that

$$
\begin{aligned}
\left\{\phi_{1}, \phi_{2}\right\}= & \lim _{n} \int \frac{d l}{l^{2}\left(1+l^{2}\right)}\left(\left(D \phi_{1}\right)_{l}-\left(D \phi_{1}\right)_{0}, \chi_{n} S_{l}\left(\frac{\left(D \phi_{2}\right)-\left(D \phi_{2}\right)_{0}}{l}\right)\right) \\
= & \lim _{n} \int \frac{d l}{l^{2}\left(1+l^{2}\right)}\left(\begin{array}{c}
2 l\left(1+l^{2}\right) r_{l} x_{l} \\
0
\end{array}\right), \chi_{n}\left(\begin{array}{cc}
0 & \frac{1}{r_{l}\left(1+l^{2}\right)} \\
-\frac{1}{r_{l}\left(1+l^{2}\right)} & 0
\end{array}\right) \\
& \cdot\left(\begin{array}{l}
\left(1+l^{2}\right) r_{l} y_{l} \\
\left(1+l^{2}\right) r_{l} x_{l}
\end{array}\right) \\
= & \lim _{n} 2 \int d l \chi_{n} \frac{r_{l} x_{l}^{2}}{l} \\
= & 2\left(\phi_{1}+1\right) .
\end{aligned}
$$

The other brackets are similar. Also, if

$$
F=\int h_{k} x_{k} d k \text { and } G=\int g_{k} y_{k} d k \text { with } h, g \in \mathscr{C}_{0}^{\infty}(\mathbb{R}),
$$

then

$$
\{F, G\}=\int \frac{l h_{l} g_{l}}{r_{l}} d l .
$$

Letting $h$ and $g$ converge to delta function, we obtain formally

$$
\left\{x_{k}, y_{l}\right\}=\delta(k-l) \frac{l}{r_{l}}
$$

which is useful for computations.

We now resume the discussion of $M$. As $r_{k}>0$ we can define a smooth phase $\theta(k ; q)$ and make the following definition:

$$
\begin{aligned}
x_{k} & \equiv \sqrt{\frac{i}{\pi}} e^{i \theta(k ; q) / 2} f_{k}(0 ; q) \\
y_{k} & \equiv \sqrt{\frac{i}{\pi}} e^{i \theta(k ; q) / 2} f_{k}^{\prime}(0 ; q) \\
\text { for }-\infty & <k<\infty .
\end{aligned}
$$


Theorem 1. The map $M \ni q \rightarrow\left(x_{k}, y_{k}\right) \in \mathscr{H}_{1}$ is continuous and to one. Also, translation $q(\cdot+t)$ becomes

$$
\begin{aligned}
& \frac{d}{d t} x_{k}=y_{k} \\
& \frac{d}{d t} y_{k}=\left[\int\left(l r_{l} x_{l}^{2}-\frac{r_{l} y_{l}^{2}}{l}\right) d l\right] x_{k}-k^{2} x_{k}
\end{aligned}
$$

for $-\infty<k<\infty$.

Proof. As $f_{k}^{\prime}(0 ; q)$ and $f_{k}^{\prime}(0 ; q)$ are smooth and $f_{k}(0 ; q)$ is bounded while $f_{k}^{\prime}(0 ; q)$ grows at most linearly, $\left(\sqrt{\frac{i}{\pi}} e^{i \theta(k ; q) / 2} f_{k}(0 ; q), \sqrt{\frac{i}{\pi}} e^{i \theta(k ; q) / 2} f_{k}^{\prime}(0, q)\right)$ is in $\mathscr{H}_{1}$ and the continuous dependence on $q$ is easily verified.

Upon translation $q(\cdot) \rightarrow q(\cdot+t)$

$$
\begin{aligned}
& f_{k}(0 ; q(\cdot+t))=e^{-i k t} f_{k}(t ; q) \\
& f_{k}^{\prime}(0 ; q(\cdot+t))=e^{-i k t} f_{k}^{\prime}(t ; q) \\
& R(k ; q(\cdot+t))=e^{2 i k t} R(k ; q)
\end{aligned}
$$

so that

$$
\begin{aligned}
& x_{k}(t)=\sqrt{\frac{i}{\pi}} e^{i \theta(k ; q) / 2} f_{k}(t ; q) \\
& y_{k}(t)=\sqrt{\frac{i}{\pi}} e^{i \theta(k ; q) / 2} f_{k}^{\prime}(t ; q) .
\end{aligned}
$$

The system (4) $)_{k}$ is now obtained by substituting (2) in the Schrödinger equation. An elementary modification of the methods of Sect. 4, Deift and Trubowitz [3a], shows that $(4)_{k}$ satisfies a Lipschitz condition on $\mathscr{H}_{1}$. Hence the map is one to one.

The point is that the equations $(4)_{k}$ are obtained ${ }^{8}$ by constraining $\mathscr{H}_{0}$ to $X$ $=\left\{\phi_{1}=0, \phi_{2}=0\right\}$ as in the example of Sect. 2. Here it is easy to check directly that

$$
\begin{aligned}
\{F, G\}_{x} & \equiv\{F, G\}-\left(\left\{F, \phi_{1}\right\},\left\{F, \phi_{2}\right\}\right)\left(\begin{array}{cc}
0 & \frac{1}{\left\{\phi_{2}, \phi_{1}\right\}} \\
\frac{1}{\left\{\phi_{1}, \phi_{2}\right\}} & 0
\end{array}\right)\left(\begin{array}{l}
\left\{\phi_{1}, G\right\} \\
\left\{\phi_{2}, G\right\}
\end{array}\right) \\
& =\{F, G\}+\frac{1}{2}\left(\left\{F, \phi_{1}\right\}\left\{\phi_{2}, G\right\}-\left\{F, \phi_{2}\right\}\left\{\phi_{1}, G\right\}\right)
\end{aligned}
$$

for $F, G \in \mathscr{A}$, is the appropriate Poisson structure on $X$.

As in the finite dimensional case, the motion is integrable. Let $\mathscr{B}$ denote the class of smooth, even functions on $\mathbb{R}$ which, together with their derivatives, are polynomially bounded.

8 Strictly speaking $(4)_{k}$ are obtained only in the weak sense as $F(z) \equiv x_{k} \notin \mathscr{A}$ and the Poisson brackets $\left\{x_{k}, G\right\}$ must be smeared 
Theorem 2. For all $b, b^{\prime} \in \mathscr{B}$,

$$
\begin{aligned}
& A_{1}(b)=\int \frac{b_{k} r_{k} x_{k}^{2}}{k} d k+\frac{1}{2} \iint \frac{b_{k}-b_{l}}{k^{2}-l^{2}} \frac{r_{k} r_{l}}{k l}\left(x_{k} y_{l}-x_{l} y_{k}\right)^{2} d k d l \\
& A_{2}(b)=\int \frac{b_{k} r_{k}}{k}\left(x_{k} y_{-k}-x_{-k} y_{k}\right) d k
\end{aligned}
$$

are in $\mathscr{A}$, and

$$
\left\{A_{i}(b), A_{j}\left(b^{\prime}\right)\right\}=\left\{A_{i}(b), A_{j}\left(b^{\prime}\right)\right\}_{X}=0, \quad i, j=1,2 .
$$

Moreover,

$$
\begin{aligned}
A_{1}(1) & =\phi_{1}+1 \\
& =1 \text { on } X
\end{aligned}
$$

and

$$
\begin{aligned}
A_{1}\left(k^{2}\right)= & \int k r_{k} x_{k}^{2} d k+\left(\int \frac{r_{k} x_{k}^{2}}{k} d k\right)\left(\int \frac{r_{k} y_{k}^{2}}{k} d k\right) \\
& -\left(\int \frac{r_{k} x_{k} y_{k}}{k} d k\right)^{2} \\
= & 2 H_{0} \text { on } X .
\end{aligned}
$$

The proof of this theorem, which we omit, is a simple modification of the finite dimensional case. The functions $A_{j}(b)$ are defined as suitable principal values and one easily verifies that $A_{j}(b) \in \mathscr{A}$. Formally

$$
A_{1}(b)=\int b_{k} A_{k} d k
$$

where

$$
A_{k}=\frac{r_{k} x_{k}^{2}}{k}+\int \frac{\left(x_{k} y_{l}-x_{l} y_{k}\right)^{2}}{k^{2}-l^{2}}\left[\frac{r_{k} r_{l}}{k l}\right] d l .
$$

Clearly $A_{k}$ is not well defined because the integral does not exist on $\mathscr{H}_{1}$, and further, $r_{k} x_{k}^{2} / k$ has only a distributional gradient. But these problems are eliminated by smearing with $b$ 's (cf. footnote 8 ).

Similar considerations apply to $A_{2}(b)$. Here $A_{2}$ 's are needed as well as $A_{1}$ 's because of an inherent symmetry. We illustrate this with a finite dimensional example. Let $H_{0}=\frac{1}{2} \sum_{i= \pm 1, \ldots . \pm m}\left(a_{i} x_{i}^{2}+y_{i}^{2}\right)$ be a finite dimensional system with $n=2 m$ degrees of freedom. If the $a_{j}$ 's are distinct then

$$
A_{k}=x_{k}^{2}+\sum_{l \neq k} \frac{\left(x_{k} y_{l}-x_{l} y_{k}\right)^{2}}{a_{k}-a_{l}}, \quad k= \pm 1, \ldots, \pm m
$$

are the conserved quantities for $H_{0}$ restricted to

$$
X=\left\{\sum_{i= \pm 1, \ldots, \pm m} x_{i}^{2}=1, \sum_{i= \pm 1, \ldots, \pm m} x_{i} y_{i}=0\right\} .
$$


If $a_{j}=a_{-j}, j=1, \ldots, m$, the individual $A_{k}$ 's are no longer defined and must be symmetrized, i.e.,

$$
\begin{array}{rlrl}
A_{1, k} \equiv & x_{k}^{2}+x_{-k}^{2}+\sum_{l \neq k,-k} \frac{\left(x_{k} y_{l}-x_{l} y_{k}\right)^{2}}{a_{k}-a_{l}} & \\
& +\sum_{l \neq k,-k} \frac{\left(x_{-k} y_{l}-x_{l} y_{-k}\right)^{2}}{a_{k}-a_{l}}, \quad k=1, \ldots, m \\
A_{2, k} \equiv & x_{k} y_{-k}-x_{-k} y_{k}, & k=1, \ldots, m .
\end{array}
$$

Of course

$$
A_{1, k}=\lim _{\substack{a_{j} \rightarrow a_{-j} \\ j=1, \ldots, m}} \mathrm{~A}_{k}+\mathrm{A}_{-k}
$$

and

$$
A_{2, k}=\lim _{\substack{a_{j} \rightarrow a_{-i} \\ j=1, \ldots, m}}\left(a_{k}-a_{-k}\right) A_{k}
$$

so that the $2 m$ functions $A_{j, k}, j=1,2, k=1, \ldots, m$, Poisson commute. In the infinite dimensional case $k^{2}=l^{2}$ for $k=l$ or $k=-l$ and $A_{1}(b)$ and $A_{2}(b)$ are simply the analogs of $A_{1, k}$ and $A_{2, k}$.

To summarize, we now have $M$ imbedded in $\mathscr{H}_{1}$ and have identified the flow of translation on $M$ with the restriction of constrained harmonic motion $A\left(k^{2}\right)$. We will now show that under the imbedding $\mathrm{KdV}$ can be identified with the restriction of $2 A\left(k^{4}\right)$.

If $q(x, t)$ solves the $\mathrm{KdV}$ equation

$$
q_{t}-6 q q_{x}+q_{x x x}=0, \quad q(x, 0)=q_{0}(x)
$$

then (see Lax [5]), $H(t) \equiv-d^{2} / d x^{2}+q(x, t)$ solves

$$
\frac{d}{d t} H=B H-H B
$$

where $B=-4 d^{3} / d x^{3}+3(q(x, t)(d / d x)+(d / d x) q(x, t))$. It follows that

$$
\begin{aligned}
\frac{d}{d t} f_{k}(x, t)= & -4 f_{k}^{\prime \prime \prime}(x, t)+6 q(x, t) f_{k}^{\prime}(x, t)+3 q^{\prime}(x, t) f_{k}(x, t) \\
& +4(i k)^{3} f_{k}(x, t)
\end{aligned}
$$

or

$$
\begin{aligned}
\frac{d}{d t} f(x, t)= & f(x, t)\left(4(i k)^{3}-q^{\prime}(x, t)\right)+f^{\prime}(x, t)\left(2 q(x, t)+4 k^{2}\right) \\
\frac{d}{d t} f^{\prime}(x, t)= & f(x, t)\left(2 q^{2}(x, t)+2 k^{2} q(x, t)-4 k^{4}-q^{\prime \prime}(x, t)\right) \\
& +f^{\prime}(x, t)\left(q^{\prime}(x, t)+4(i k)^{3}\right),
\end{aligned}
$$


where second derivatives have been eliminated by $f_{k}^{\prime \prime}(x, t)=\left(q(x, t)-k^{2}\right) f_{k}(x, t)$. Also $R(k ; q(\cdot, t))=e^{8 i k^{3} t} R\left(k ; q_{0}\right)$ so that

$$
\begin{aligned}
x_{k}(t) & \equiv \sqrt{\frac{i}{\pi}} e^{i \theta(k ; q(\cdot, t)) / 2} f_{k}(0, t) \\
& =\sqrt{\frac{i}{\pi}} e^{i \theta\left(k ; q_{0}\right) / 2}\left(e^{4 i k^{3} t} f_{k}(0, t)\right), \\
y_{k}(t) & \equiv \sqrt{\frac{i}{\pi}} e^{i \theta(k ; q(\cdot, t)) / 2} f_{k}^{\prime}(0, t) \quad-\infty<k<\infty, \\
& =\sqrt{\frac{i}{\pi}} e^{i \theta\left(k ; q_{0}\right) / 2}\left(e^{4 i k^{3} t} f_{k}^{\prime}(0, t)\right),
\end{aligned}
$$

satisfy

$$
\begin{aligned}
& \frac{d}{d t} x_{k}(t)=-q^{\prime}(0, t) x_{k}(t)+\left(2 q(0, t)+4 k^{2}\right) y_{k}(t) \\
& \frac{d}{d t} y_{k}(t)=\left(2 q^{2}(0, t)+2 k^{2} q(0, t)-q^{\prime \prime}(0, t)-4 k^{4}\right) x_{k}(t)+q^{\prime}(0, t) y_{k}(t) .
\end{aligned}
$$

From (1) and (3)

$$
q^{\prime}(x)=\frac{4 i}{\pi} \int k R(k ; q) f_{k}(x ; q) f_{k}^{\prime}(x ; q) d k
$$

and

$$
\begin{aligned}
q^{\prime \prime}(x)= & \frac{4 i}{\pi} \int k R(k ; q)\left(f_{k}(x ; q) f_{k}^{\prime \prime}(x ; q)+\left(f_{k}^{\prime}(x ; q)\right)^{2}\right) d k \\
= & \frac{4 i q(x)}{\pi} \int k R(k ; q) f_{k}^{2}(x ; q) d k \\
& -\frac{4 i}{\pi} \int k^{3} R(k ; q) f_{k}^{2}(x ; q) d k+\frac{4 i}{\pi} \int k R(k ; q)\left(f_{k}^{\prime}(x ; q)\right)^{2} d k
\end{aligned}
$$

At $x=0$ this becomes

$$
\begin{aligned}
q^{\prime}(0, t) & =4 \int k r_{k} x_{k}(t) y_{k}(t) d k \\
q^{\prime \prime}(0, t)-2 \dot{q}^{2}(0, t) & =-4 \int k^{3} r_{k} x_{k}^{2}(t) d k+4 \int k r_{k} y_{k}^{2}(t) d k .
\end{aligned}
$$

On the other hand,

$$
\begin{aligned}
2 A\left(k^{4}\right)= & 2 \int k^{3} r_{k} x_{k}^{2} d k \\
& +2\left(\int k r_{k} x_{k}^{2} d k\right)\left(\int \frac{r_{k} y_{k}^{2}}{k} d k\right)+2\left(\int \frac{r_{k} x_{k}^{2}}{k} d k\right)\left(\int k r_{k} y_{k}^{2} d k\right) \\
& +4\left(\int k r_{k} x_{k} y_{k}\right)\left(\int \frac{r_{k}}{l_{r}} x_{k} y_{k}\right)
\end{aligned}
$$


which reduces to

$$
H_{1}=2 \int k^{3} r_{k} x_{k}^{2} d k+2 \int k r_{k} y_{k}^{2}+2\left(\int k r_{k} x_{k}^{2} d k\right)\left(\int \frac{r_{k} y_{k}^{2}}{k} d k\right)
$$

on

$$
\mathrm{X}=\left\{\phi_{1}=\int \frac{r_{k} x_{k}^{2}}{k} d k-1=0, \phi_{2}=\int \frac{r_{k} x_{k} y_{k}}{k} d k=0\right\} .
$$

Choosing $\alpha_{1}$ and $\alpha_{2}$ so that $\left\{\phi_{1}, H_{1}+\alpha_{1} \phi_{1}+\alpha_{2} \phi_{2}\right\}=0=\left\{\phi_{2}, H_{1}+\alpha_{1} \phi_{1}+\alpha_{2} \phi_{2}\right\}$ on $X$, we find

$$
\begin{aligned}
& \alpha_{1}=2 \int\left(k r_{k} y_{k}^{2}-k^{3} r_{k} x_{k}^{2}\right) d k \\
& \alpha_{2}=-4 \int k r_{k} x_{k} y_{k},
\end{aligned}
$$

and $2 A\left(k^{4}\right)$ constrainted to $X$ becomes (cf. footnote 8 )

$$
\begin{aligned}
\frac{d}{d t} x_{k} & =\left\{x_{k}, H_{1}+\alpha_{1} \phi_{1}+\alpha_{2} \phi_{2}\right\} \\
& =\left(4 k^{2}+4\left(\int l r_{l} x_{l}^{2} d l\right)\right) y_{k}-4\left(\int l r_{l} x_{l} y_{l} d l\right) x_{k} \\
\frac{d}{d t} y_{k} & =\left\{y_{k}, H_{1}+\alpha_{1} \phi_{1}+\alpha_{2} \phi_{2}\right\} \\
& =4\left(\int l r_{l} x_{l} y_{l} d l\right) y_{k}+\left(4 \int\left(l^{3} r_{l} x_{l}^{2}-l r_{l} y_{l}^{2}\right) d l-4 k^{2} \int \frac{r_{l} y_{l}^{2}}{l} d l-4 k^{4}\right) x_{k} .
\end{aligned}
$$

But by (6) and $q(0, t)=2 \int l r_{l} x_{l}^{2} d l=-2 \int \frac{r_{l} y_{l}^{2}}{l} d l$, these equations are identical to $(5)_{k}$. This identifies $\mathrm{KdV}$ with the restriction of $2 A\left(k^{4}\right)$.

Finally, $\left\{A\left(k^{2}\right), A\left(k^{4}\right)\right\}_{X}=0$ so the study of $\mathrm{KdV}$ reduces to the study of an integrable system of constrained harmonic oscillators. The same is true for $A\left(k^{2 n}\right)$, $n=2,3, \ldots$, which correspond to the higher order $\mathrm{KdV}$ flows.

In [3b], it is shown that $M$ is in general a product of lines and circles. When $r_{k}>0$, as above, $M$ is a product of lines. Otherwise we must add one circle each time $r_{k}$ vanishes. When $M$ is not simply connected, it it possible to define a phase only on the product of lines. However, this is enough for our purposes because all the $\mathrm{KdV}$ vector fields are transverse to the toroidal piece of $M$.

\section{The Nonlinear Schrödinger Equation}

The nonlinear Schrödinger equation

$$
i \frac{\partial q}{\partial t}+\frac{\partial^{2} q}{\partial x^{2}}+|q|^{2} q=0
$$


where $q \rightarrow 0$ as $x \rightarrow \pm \infty$ describes the evolution of the envelope of an almost monochromatic wave. We will show how to reduce the study of this equation to that of integrable constrained harmonic motion. In the last section all the constructions and calculations were rigorous. Here, for simplicity, some things will be formal even though the techniques introduced for the KdV equation can be applied with minor changes.

Let $q, r$ be complex valued functions vanishing ar $\pm \infty$ and let $^{9} \psi_{+}(x, k)$, $\phi_{+}(x, k), \psi_{-}(x, k)$, and $\phi_{-}(x, k)$ be solutions of

$$
\begin{aligned}
& v_{1}^{\prime}+i k v_{1}=q v_{2} \\
& v_{2}^{\prime}-i k v_{2}=r v_{1}
\end{aligned}
$$

with asymptotics

$$
\begin{gathered}
\psi_{+}=\left(\begin{array}{l}
\psi_{+1} \\
\psi_{+2}
\end{array}\right)_{x \rightarrow+\infty}\left(\begin{array}{l}
0 \\
1
\end{array}\right) e^{i k x}, \\
\phi_{+}=\left(\begin{array}{l}
\phi_{+1} \\
\phi_{+2}
\end{array}\right)_{x \rightarrow-\infty}\left(\begin{array}{l}
1 \\
0
\end{array}\right) e^{-i k x}, \\
\psi_{-}=\left(\begin{array}{l}
\psi_{-1} \\
\psi_{-2}
\end{array}\right)_{x \rightarrow+\infty}\left(\begin{array}{l}
1 \\
0
\end{array}\right) \mathrm{e}^{-i k x},
\end{gathered}
$$

and

$$
\phi_{-}=\left(\begin{array}{l}
\phi_{-1} \\
\phi_{-2}
\end{array}\right)_{x \rightarrow-\infty} \sim\left(\begin{array}{r}
0 \\
-1
\end{array}\right) e^{i k x} .
$$

The solutions $\psi_{+}, \phi_{+}$are analytic in the upper half $k$-plane while $\psi_{-}, \phi_{-}$are analytic in the lower half $k$-plane. They are related by the transition matrix

$$
\begin{gathered}
\left(\begin{array}{cc}
a_{+}(k) & b_{+}(k) \\
a_{-}(k) & b_{-}(k)
\end{array}\right) \text { through } \\
\psi_{+}=-a_{+} \phi_{-}+b_{-} \phi_{+}, \\
\phi_{+}=a_{+} \psi_{-}+b_{+} \psi_{+}, \\
\psi_{-}=a_{-} \phi_{+}+b_{+} \phi_{-},
\end{gathered}
$$

and

$$
\phi_{-}=-a_{-} \psi_{+}+b_{-} \psi_{-} .
$$

The functions $a_{+}, a_{-}$are analytic in the upper and lower half $k$-planes respectively. To simplify the presentation we will assume that both $a_{+}$and $a_{-}$are root free. Set

$$
r_{+}(k)=b_{+}(k) / a_{+}(k) \text { and } r_{-}(k)=b_{-}(k) / a_{-}(k) \text {. }
$$

9 See Ablowitz et al. [1a] for the basic spectral theory 


\section{Lemma 1}

(i) $q(x)=-\frac{1}{\pi} \int_{-\infty}^{\infty} r_{+}(k) \psi_{+1}^{2}(x, k)+r_{-}(k) \psi_{-1}^{2}(x, k) d k$

(ii) $\quad r(x)=\frac{1}{\pi} \int_{-\infty}^{\infty} r_{+}(k) \psi_{+2}^{2}(x, k)+r_{-}(k) \psi_{-2}^{2}(x, k) d k$

(iii) $i \pi\left(r_{-}(0) \psi_{-1}^{2}(x, 0)-2 \psi_{+1}(x, 0) \psi_{-1}(x, 0)-r_{+}(0) \psi_{+1}^{2}(x, 0)\right)$

$$
+\int_{-\infty}^{\infty} \frac{r_{+}(k)}{k} \psi_{+1}^{2}(x, k)+\frac{r_{-}(k)}{k} \psi_{-1}^{2}(x, k) d k=0
$$

(iv) $i \pi\left(r_{-}(0) \psi_{-2}^{2}(x, 0)-2 \psi_{+2}(x, 0) \psi_{-2}(x, 0)-r_{+}(0) \psi_{+2}^{2}(x, 0)\right)$

$$
+\int_{-\infty}^{\infty} \frac{r_{+}(k)}{k} \psi_{+2}^{2}(x, k)+\frac{r_{-}(k)}{k} \psi_{-2}^{2}(x, k) d k=0
$$

(v) $i \pi\left(r_{-}(0) \psi_{-1}(x, 0) \psi_{-2}(x, 0)-\psi_{+1}(x, 0) \psi_{-2}(x, 0)\right.$

$$
\begin{aligned}
& \left.-\psi_{-1}(x, 0) \psi_{+2}(x, 0)-r_{+}(0) \psi_{+1}(x, 0) \psi_{+2}(x, 0)\right) \\
& +\int_{-\infty}^{\infty} \frac{r_{+}(k)}{k} \psi_{+1}(x, k) \psi_{+2}(x, k)+\frac{r_{-}(k)}{k} \psi_{-1}(x, k) \psi_{-2}(x, k) d k \\
= & -\pi i .
\end{aligned}
$$

Proof. (i) and (ii) are obtained just as in Sect. 3. The derivation of the remaining formulae is very similar so that we will only prove (iii). We have

$$
\frac{\phi_{+1}(x, k) \psi_{+1}(x, k)}{k a_{+}(k)}=\frac{q(x)}{2 i k^{2}}+O\left(\frac{1}{k^{3}}\right)
$$

when $\operatorname{Im} k \geqq 0,|k| \rightarrow+\infty$. Now integrate around the appropriate contour in $\operatorname{Im} k \geqq 0$ to find

$$
\begin{aligned}
0= & \int_{-\infty}^{\infty} \frac{\phi_{+1}(x, k) \psi_{-1}(x, k)}{k a_{+}} d k-\pi i \frac{\phi_{+1}(x, 0) \psi_{+1}(x, 0)}{a_{+}(0)} \\
= & \int_{-\infty}^{\infty} \frac{r_{+}(k) \psi_{+1}^{2}(x, k)+\psi_{+1}(x, k) \psi_{-1}(x, k)}{k} d k \\
& -\pi i\left(\psi_{+1}(x, 0) \psi_{-1}(x, 0)+r_{+}(0) \psi_{+1}^{2}(x, 0)\right) .
\end{aligned}
$$

Similarly,

$$
\begin{aligned}
0= & \int_{-\infty}^{\infty} \frac{r_{-}(k) \psi_{-1}^{2}(x, k)+\psi_{-1}(x, k) \psi_{+1}(x, k)}{k} d k \\
& +\pi i\left(r_{-}(0) \psi_{-1}^{2}(x, 0)-\psi_{+1}(x, 0) \psi_{-1}(x, 0)\right) .
\end{aligned}
$$

Formula (iii) follows immediately.

The quadratic form

$$
Q=i r_{-}(0) \psi_{-}^{2}(x, 0)-2 i \psi_{+1}(x, 0) \psi_{-1}(x, 0)-i r_{+}(0) \psi_{+}^{2}(x, 0)
$$


can always be brought to principal axes

$$
Q=\left(c_{11} \psi_{-1}(x, 0)+c_{12} \psi_{+1}(x, 0)\right)^{2}+\left(c_{21} \psi_{-1}(x, 0)+c_{22} \psi_{+1}(x, 0)\right)^{2}
$$

because

$$
\begin{aligned}
\left|\begin{array}{cc}
-i r_{+}(0) & -i \\
-i & i r_{-}(0)
\end{array}\right| & =\left(1+r_{+}(0) r_{-}(0)\right) \\
& =\left(1+\frac{b_{+}(0) b_{-}(0)}{a_{+}(0) a_{-}(0)}\right) \\
& =\frac{1}{a_{+}(0) a_{-}(0)} \neq 0 .
\end{aligned}
$$

In the last line we have used the identity $a_{+}(k) a_{-}(k)+b_{+}(k) b_{-}(k)=1$ and the fact that both $a_{+}(0)$ and $a_{-}(0)$ are finite. Set

$$
\begin{array}{ll}
x_{+k}=\sqrt{\frac{r_{+}(k)}{\pi k}} \psi_{+1}(0, k), & x_{-k}=\sqrt{\frac{r_{-}(k)}{\pi k}} \psi_{-1}(0, k) \\
y_{+k}=\sqrt{\frac{r_{+}(k)}{\pi k}} \psi_{+2}(0, k), \quad y_{-k}=\sqrt{\frac{r_{-}(k)}{\pi k}} \psi_{-2}(0, k)
\end{array}
$$

for all $k \neq 0$ and

$$
\begin{aligned}
& x_{+0}=c_{11} \psi_{-1}(0,0)+c_{12} \psi_{+1}(0,0) \\
& x_{-0}=c_{21} \psi_{-1}(0,0)+c_{22} \psi_{+1}(0,0) \\
& y_{+0}=c_{11} \psi_{-2}(0,0)+c_{12} \psi_{+2}(0,0) \\
& y_{-0}=c_{21} \psi_{-2}(0,0)+c_{22} \psi_{+2}(0,0) .
\end{aligned}
$$

It is easy to see that under the map

$$
(q, r) \rightarrow\left(x_{+k}, y_{+k}, x_{-k}, y_{-k} ;-\infty<k<\infty\right)
$$

translation becomes

$$
\begin{aligned}
& \frac{d}{d t} x_{+k}=-i k x_{+k}-\left[\int_{-\infty}^{\infty} l\left(x_{+l}^{2}+x_{-l}^{2}\right) d l\right] y_{+k} \\
& \frac{d}{d t} y_{+k}=i k y_{+k}+\left[\int_{-\infty}^{\infty} l\left(y_{+l}^{2}+y_{-l}^{2}\right) d l\right] x_{+k} \\
& \frac{d}{d t} x_{-k}=-i k x_{-k}-\left[\int_{-\infty}^{\infty} l\left(x_{+l}^{2}+x_{-l}^{2}\right) d l\right] y_{-k} \\
& \frac{d}{d t} y_{-k}=i k y_{-k}+\left[\int_{-\infty}^{\infty} l\left(y_{+l}^{2}+y_{-l}^{2}\right) d l\right] x_{-k} .
\end{aligned}
$$


Let

$$
\begin{aligned}
& \phi_{1}=x_{+0}^{2}+x_{-0}^{2}+\int_{-\infty}^{\infty} x_{+k}^{2}+x_{-k}^{2} d k, \\
& \phi_{2}=y_{+0}^{2}+y_{-0}^{2}+\int_{-\infty}^{\infty} y_{+k}^{2}+y_{-k}^{2} d k, \\
& \phi_{3}=x_{+0} y_{+0}+x_{-0} y_{-0}+\int_{-\infty}^{\infty} x_{+k} y_{+k}+x_{-k} y_{-k} d k,
\end{aligned}
$$

and

$$
H_{0}=-\int_{-\infty}^{\infty} i k\left(x_{+k} y_{+k}+x_{-k} y_{-k}\right)
$$

In the Poisson structure

$$
\begin{aligned}
\left\{x_{+k}, y_{+l}\right\} & =\left\{x_{-k}, y_{-l}\right\}=\delta_{k l} \\
\left\{x_{+k}, x_{-l}\right\} & =\left\{y_{+k}, y_{-l}\right\}=\left\{x_{+k}, y_{-l}\right\}=\left\{x_{-k}, y_{+l}\right\}=0, \\
\left\{\phi_{1}, \phi_{2}\right\} & =4 \phi_{3} \\
\left\{\phi_{1}, H_{0}\right\} & =-2 i \int_{-\infty}^{\infty} k\left(x_{+k}^{2}+x_{-k}^{2}\right) d k,
\end{aligned}
$$

and

$$
\left\{\phi_{2}, H_{0}\right\}=2 i \int_{-\infty}^{\infty} k\left(y_{+k}^{2}+y_{-k}^{2}\right) d k .
$$

Constraining $H_{0}$ to $X=\left\{\phi_{1}=\phi_{2}=0\right\}$ we find $H=H_{0}+\alpha_{1} \phi_{1}+\alpha_{2} \phi_{2}$ where $\alpha_{1}, \alpha_{2}$ are determined by

$$
\begin{aligned}
& 0=\left\{\phi_{1}, H\right\}=-2 i \int_{-\infty}^{\infty} k\left(x_{+k}^{2}+x_{-k}^{2}\right) d k+4 \alpha_{2} \phi_{3}, \\
& 0=\left\{\phi_{2}, H\right\}=2 i \int_{-\infty}^{\infty} k\left(y_{+k}^{2}+y_{-k}^{2}\right)+4 \alpha_{1} \phi_{3}
\end{aligned}
$$

on $X$ so that

$$
\begin{aligned}
\alpha_{1} & =\frac{-i \int_{-\infty}^{\infty} k\left(y_{+k}^{2}+y_{-k}^{2}\right) d k}{2 \phi_{3}} \\
\alpha_{2} & =\frac{i \int_{-\infty}^{\infty} k\left(x_{+k}^{2}+x_{-k}^{2}\right)}{2 \phi_{3}} .
\end{aligned}
$$


The equations of constrained motion become

$$
\begin{aligned}
& \frac{d}{d t} x_{+k}=\left\{x_{+k}, H\right\}=-i k x_{+k}+\left[\frac{i \int_{-\infty}^{\infty} k\left(x_{+k}^{2}+x_{-k}^{2}\right) d k}{\phi_{3}}\right] y_{+k} \\
& \frac{d}{d t} y_{+k}=\left\{y_{+k}, H\right\}=i k y_{+k}+\left[\frac{-i \int_{-\infty}^{\infty} k\left(y_{+k}^{2}+y_{-k}^{2}\right)}{\phi_{3}}\right] x_{+k} \\
& \frac{d}{d t} x_{-k}=\left\{x_{-k}, H\right\}=-i k x_{-k}+\left[\frac{i \int_{-\infty}^{\infty} k\left(x_{+k}^{2}+x_{-k}^{2}\right) d k}{\phi_{3}}\right] y_{-k} \\
& \frac{d}{d t} y_{-k}=\left\{y_{-k}, H\right\}=i k y_{-k}+\left[\frac{-i \int_{-\infty}^{\infty} k\left(y_{+k}^{2}+y_{-k}^{2}\right) d k}{\phi_{3}}\right] x_{-k} .
\end{aligned}
$$

$-\infty<k<\infty$.

But $\left\{\phi_{3}, H\right\}=0$ on $X$ so that $\phi_{3}$ is conserved for the constrained motion. Thus system (1) is obtained by constraining $H_{0}$ to $X$ and restricting to the hypersurface $\phi_{3}=-i$. This is consistent with Lemma 1 , (v). Moreover, the functions

$$
\begin{aligned}
B_{+k}= & \frac{\left(x_{+k} y_{+0}-x_{+0} y_{+k}\right)^{2}+\left(x_{+k} y_{-0}-x_{-0} y_{+k}\right)^{2}}{k} \\
& +\int_{-\infty}^{\infty} \frac{\left(x_{+k} y_{+l}-x_{+l} y_{+k}\right)^{2}+\left(x_{+k} y_{-l}-x_{-l} y_{+k}\right)^{2}}{k-l} d l \\
B_{-k}= & \frac{\left(x_{-k} y_{+0}-x_{+0} y_{-k}\right)^{2}+\left(x_{-k} y_{-0}-x_{-0} y_{-k}\right)^{2}}{k} \\
& +\int_{-\infty}^{\infty} \frac{\left(x_{-k} y_{+l}-x_{+l} y_{-k}\right)^{2}+\left(x_{-k} y_{-l}-x_{-l} y_{-k}\right)^{2}}{k-l} d l,
\end{aligned}
$$

$-\infty<k<\infty$, Poisson commute and are conserved under the constrained motion with the result that the system is integrable. Finally, if $r=-q^{*}$ and $q$ evolves according to the nonlinear Schrödinger equation, the time evolution of $\psi_{+}$is given by

$$
\begin{aligned}
& i \frac{d}{d t} \psi_{+1}=\left(k^{2}-|q|^{2}\right) \psi_{+1}+\left(i k q-\frac{1}{2} q_{x}\right) \psi_{+2}-2 i k^{2} \psi_{+1} \\
& i \frac{d}{d t} \psi_{+2}=-\left(k^{2}-|q|^{2}\right) \psi_{+2}-\left(i k q^{*}+\frac{1}{2} q_{x}^{*}\right) \psi_{+1}-2 i k^{2} \psi_{+2}
\end{aligned}
$$

and similarly for $\phi_{+}$. In this case

$$
\begin{aligned}
& \psi_{-}(x, k)=\left(\psi_{+2}^{*}\left(x, k^{*}\right),-\psi_{+1}^{*}\left(x, k^{*}\right)\right), \\
& \phi_{-}(x, k)=\left(\phi_{+2}^{*}\left(x, k^{*}\right),-\phi_{+1}^{*}\left(x, k^{*}\right)\right)
\end{aligned}
$$

and $a_{-}(k)=a_{+}(-k), b_{-}(k)=b_{+}(-k)$. 
Imitating the calculation at the end of the last section it is easy to chek that the nonlinear Schrödinger equation can be identified with the system obtained by constraining $\int_{-\infty}^{\infty} k^{3}\left(B_{k}+B_{-k}\right) d k$ to $X$ and restricting to $\phi_{3}=-i, x_{-k}=y_{+k}^{*}$, and $y_{-k}=-x_{+k}^{*}$. A simple calculation shows that the last condition is preserved in time.

\section{The Sine-Gordon Equation}

Here we will show how to reduce the study of the Sine-Gordon equation

$$
\frac{\partial^{2} q}{\partial t^{2}}-\frac{\partial^{2} q}{\partial x^{2}}+\sin q=0,
$$

where $q \rightarrow 2 \pi n(n \in Z)$ as $x \rightarrow \pm \infty$, to the study of integrable constrained harmonic motion. Again, for simplicity, some of the calculations will be formal.

Let $q(x, 0),(\partial / \partial t) q(x, 0)$ be real, $w_{ \pm}=\left(\frac{\partial}{\partial x} q(x, 0) \pm \frac{\partial q}{\partial t}(x, 0)\right)$ and $\operatorname{let}^{10} \psi(x, k)$, $\phi(x, k), k \neq 0$, be solutions of

$$
\left(\begin{array}{l}
z_{1} \\
z_{2}
\end{array}\right)=\left(\begin{array}{cc}
-\frac{i k}{2}+\frac{i}{8 k} \cos q & -\frac{w_{+}}{4}+\frac{i}{8 k} \sin q \\
\frac{w_{+}}{4}+\frac{i}{8 k} \sin q & \frac{i k}{2}-\frac{i}{8 k} \cos q
\end{array}\right)\left(\begin{array}{l}
z_{1} \\
z_{2}
\end{array}\right)
$$

satisfying

$$
\begin{aligned}
& \psi(x, k)=\left(\begin{array}{l}
\psi_{1}(x, k) \\
\psi_{2}(x, k)
\end{array}\right) \underset{x \rightarrow+\infty}{\sim}\left(\begin{array}{l}
0 \\
1
\end{array}\right) e^{i\left(\frac{k}{2}-\frac{1}{8 k}\right) x}, \\
& \phi(x, k)=\left(\frac{\phi_{1}(x, k)}{\phi_{2}(x, k)}\right)_{x \rightarrow-\infty}\left(\begin{array}{l}
1 \\
0
\end{array}\right) e^{-i\left(\frac{k}{2}-\frac{1}{8 k}\right) x} .
\end{aligned}
$$

The solutions $\psi, \phi$ are analytic in the upper half $k$-plane and

$$
\phi(x, k)=\left\{\begin{array}{l}
a(k)\left(\begin{array}{r}
\psi_{2}^{*}\left(x, k^{*}\right) \\
-\psi_{1}^{*}\left(x, k^{*}\right)
\end{array}\right)+b(k) \psi(x, k), \quad \operatorname{Im} k \geqq 0 \\
a(k)\left(\begin{array}{r}
\psi_{2}(x,-k) \\
-\psi_{1}(x,-k)
\end{array}\right)+b(k) \psi(x, k), \quad k \text { real } .
\end{array}\right.
$$

Also, $a(k)$ is analytic in the upper half $k$-plane with

$$
a(k) a(-k)+b(k) b(-k)=1
$$

on the axis.

For simplicity, it is assumed that $a(k)$ is root free. Set $r_{k}=\frac{b(k)}{a(k)}$.

10 See Kaup [4] for the basic spectral theory 


\section{Lemma 1}

(i) $w_{+}(x)=\frac{2}{\pi} \int_{-\infty}^{\infty} r_{l}\left(\psi_{1}^{2}(x, l)+\psi_{2}^{2}(x, l)\right) d l$

(ii) $\quad w_{-}(x)=\frac{-1}{2 \pi} \int_{-\infty}^{\infty} \frac{r_{l}}{l^{2}}\left(\psi_{1}^{2}(x, l)+\psi_{2}^{2}(x, l)\right) d l$

(iii) $\sin q(x)=\frac{i}{\pi} \int_{-\infty}^{\infty} \frac{r_{l}}{l}\left(\psi_{1}^{2}(x, l)-\psi_{2}^{2}(x, l)\right) d l$

(iv) $\cos q(x)=1-\frac{2 i}{\pi} \int_{-\infty}^{\infty} \frac{r_{l}}{l} \psi_{1}(x, l) \psi_{2}(x, l) d l$

(v) $0=\int_{-\infty}^{\infty} \frac{r_{l}}{l\left(\frac{l}{2}-\frac{1}{8 l}\right)}\left(\psi_{1}^{2}(x, l)+\psi_{2}^{2}(x, l)\right) d l$

$$
\begin{aligned}
& +2 \pi i\left(r\left(-\frac{1}{2}\right) \psi_{2}^{2}\left(x,-\frac{1}{2}\right)-2 \psi_{1}\left(x, \frac{1}{2}\right) \psi_{2}\left(x,-\frac{1}{2}\right)-r\left(\frac{1}{2}\right) \psi_{1}^{2}\left(x, \frac{1}{2}\right)\right) \\
& +2 \pi i\left(r\left(-\frac{1}{2}\right) \psi_{1}^{2}\left(x,-\frac{1}{2}\right)+2 \psi_{1}\left(x,-\frac{1}{2}\right) \psi_{2}\left(x, \frac{1}{2}\right)-r\left(\frac{1}{2}\right) \psi_{2}^{2}\left(x, \frac{1}{2}\right)\right)
\end{aligned}
$$

(vi) $0=\int_{-\infty}^{\infty} \frac{r_{l}}{\left(\frac{l}{2}-\frac{1}{8 l}\right)}\left(\psi_{1}^{2}(x, l)-\psi_{2}^{2}(x, l)\right) d l$

$$
\begin{aligned}
& +\pi i\left(r\left(-\frac{1}{2}\right) \psi_{2}^{2}\left(x,-\frac{1}{2}\right)-2 \psi_{1}\left(x, \frac{1}{2}\right) \psi_{2}\left(x,-\frac{1}{2}\right)-r\left(\frac{1}{2}\right) \psi_{1}^{2}\left(x, \frac{1}{2}\right)\right) \\
& +\pi i\left(r\left(\frac{1}{2}\right) \psi_{2}^{2}\left(x, \frac{1}{2}\right)-2 \psi_{1}\left(x,-\frac{1}{2}\right) \psi_{2}\left(x, \frac{1}{2}\right)-r\left(-\frac{1}{2}\right) \psi_{1}^{2}\left(x,-\frac{1}{2}\right)\right)
\end{aligned}
$$

$$
\text { (vii) } \begin{aligned}
-\pi i= & \int_{-\infty}^{\infty} \frac{r_{l}}{\left(\frac{l}{2}-\frac{1}{8 l}\right)} \psi_{1}(x, l) \psi_{2}(x, l) d l \\
& +\pi i\left(\psi_{1}\left(x,-\frac{1}{2}\right) \psi_{1}\left(x, \frac{1}{2}\right)-\psi_{2}\left(x,-\frac{1}{2}\right) \psi_{2}\left(x, \frac{1}{2}\right)\right. \\
& \left.-r\left(\frac{1}{2}\right) \psi_{1}\left(\frac{1}{2}\right) \psi_{2}\left(\frac{1}{2}\right)-r\left(-\frac{1}{2}\right) \psi_{1}\left(-\frac{1}{2}\right) \psi_{2}\left(-\frac{1}{2}\right)\right) .
\end{aligned}
$$

Proof. It is enough to sketch the proof of (vi). Integrate $\frac{\phi_{1} \psi_{1}}{\left(l \mp \frac{1}{2}\right) a}, \frac{\phi_{2} \psi_{2}}{\left(l \mp \frac{1}{2}\right) a}$ around
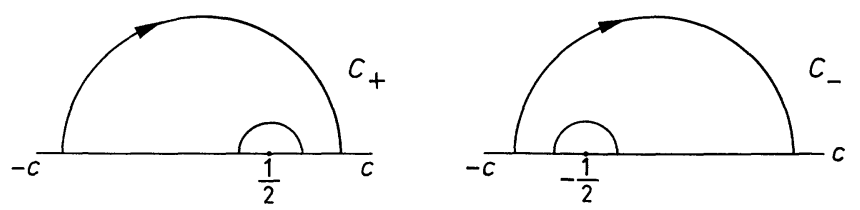

to obtain

$$
\begin{aligned}
\mathrm{I}_{ \pm}= & \int_{C_{ \pm}} \frac{\phi_{1} \psi_{1}}{\left(l \mp \frac{1}{2}\right) a} d l=-\frac{\pi i \phi_{1}\left( \pm \frac{1}{2}\right) \psi_{1}\left( \pm \frac{1}{2}\right)}{a\left( \pm \frac{1}{2}\right)} \\
& +\int_{-C}^{C} \frac{\psi_{1}(l) \psi_{2}(-l)+r_{l} \psi_{1}^{2}(l)}{\left(l \mp \frac{1}{2}\right)} d l
\end{aligned}
$$


and

$$
\begin{aligned}
\mathrm{II}_{ \pm}= & \int_{C_{ \pm}} \frac{\phi_{2} \psi_{2}}{\left(l \mp \frac{1}{2}\right) a} d l=-\frac{\pi i \phi_{2}\left( \pm \frac{1}{2}\right) \psi_{2}\left( \pm \frac{1}{2}\right)}{a\left( \pm \frac{1}{2}\right)} \\
& -\int_{-C}^{c} \frac{\psi_{1}(-l) \psi_{2}(l)+r_{l} \psi_{2}^{2}(l)}{\left(l \mp \frac{1}{2}\right)} d l
\end{aligned}
$$

Now, $\left(\mathrm{I}_{+}-\mathrm{II}_{-}\right)-\left(\mathrm{I}_{-}-\mathrm{II}_{+}\right)$

$$
\begin{aligned}
= & -\frac{\pi i \phi_{1}\left(\frac{1}{2}\right) \psi_{1}\left(\frac{1}{2}\right)}{a\left(\frac{1}{2}\right)}+\frac{\pi i \phi_{2}\left(-\frac{1}{2}\right) \psi_{2}\left(-\frac{1}{2}\right)}{a\left(-\frac{1}{2}\right)} \\
& -\frac{\pi i \phi_{1}\left(-\frac{1}{2}\right) \psi_{1}\left(-\frac{1}{2}\right)}{a\left(-\frac{1}{2}\right)}+\frac{\pi i \phi_{2}\left(\frac{1}{2}\right) \psi_{2}\left(\frac{1}{2}\right)}{a\left(\frac{1}{2}\right)} \\
& +\int_{-c}^{c} \frac{r_{l}}{\left(\frac{l}{2}-\frac{1}{8 l}\right)}\left(\psi_{1}^{2}(l)-\psi_{2}^{2}(l)\right) d l .
\end{aligned}
$$

Formula (vi) is obtained by letting $c \rightarrow \infty$ and using

$$
\phi(x, k)=a(k)\left(\begin{array}{r}
\psi_{2}(x,-k) \\
-\psi_{1}(x,-k)
\end{array}\right)+b(k) \psi(x, k) .
$$

The quadratic form

$$
\begin{aligned}
& 2 i\left(r\left(-\frac{1}{2}\right) \psi_{2}^{2}\left(x,-\frac{1}{2}\right)-2 \psi_{1}\left(x, \frac{1}{2}\right) \psi_{2}\left(x,-\frac{1}{2}\right)-r\left(\frac{1}{2}\right) \psi_{1}^{2}\left(x, \frac{1}{2}\right)\right) \\
+ & 2 i\left(r\left(-\frac{1}{2}\right) \psi_{1}^{2}\left(x,-\frac{1}{2}\right)+2 \psi_{1}\left(x,-\frac{1}{2}\right) \psi_{2}\left(x, \frac{1}{2}\right)-r\left(\frac{1}{2}\right) \psi_{2}^{2}\left(x, \frac{1}{2}\right)\right)
\end{aligned}
$$

can be written as

$$
\begin{gathered}
\left(c_{11} \psi_{1}\left(x, \frac{1}{2}\right)+c_{12} \psi_{2}\left(x,-\frac{1}{2}\right)\right)^{2}+\left(c_{21} \psi_{1}\left(x, \frac{1}{2}\right)+c_{22} \psi_{2}\left(x,-\frac{1}{2}\right)\right)^{2} \\
+\left(-c_{12} \psi_{1}\left(x,-\frac{1}{2}\right)+c_{11} \psi_{2}\left(x, \frac{1}{2}\right)\right)^{2}+\left(c_{22} \psi_{1}\left(x,-\frac{1}{2}\right)-c_{21} \psi_{2}\left(x, \frac{1}{2}\right)\right)^{2}
\end{gathered}
$$

because $^{11}$

$$
\left|\begin{array}{cc}
-r\left(\frac{1}{2}\right) & -1 \\
-1 & r\left(-\frac{1}{2}\right)
\end{array}\right|=\frac{-1}{a\left(\frac{1}{2}\right) a\left(-\frac{1}{2}\right)} \neq 0 .
$$

Set

$$
\begin{aligned}
& x_{k}=\left(\frac{r_{k}}{\pi k\left(\frac{k}{2}-\frac{1}{8 k}\right)}\right)^{1 / 2} \psi_{1}(0, k) \\
& y_{k}=\left(\frac{r_{k}}{\pi k\left(\frac{k}{2}-\frac{1}{8 k}\right)}\right)^{1 / 2} \psi_{2}(0, k)
\end{aligned}
$$

$11 a(k)$ is bounded on the axis when $q \underset{ \pm \infty}{\longrightarrow} 2 \pi n, q_{t}, q_{x} \underset{ \pm \infty}{\longrightarrow} 0$ fast enough 
for all $k \neq \pm \frac{1}{2}$ and

$$
\begin{aligned}
& x_{1 / 2}=c_{11} \psi_{1}\left(0, \frac{1}{2}\right)+c_{12} \psi_{2}\left(0,-\frac{1}{2}\right), \quad x_{-1 / 2}=c_{22} \psi_{1}\left(0,-\frac{1}{2}\right)-c_{21} \psi_{2}\left(0, \frac{1}{2}\right) \\
& y_{1 / 2}=-c_{12} \psi_{1}\left(0,-\frac{1}{2}\right)+c_{11} \psi_{2}\left(0, \frac{1}{2}\right), \quad y_{-1 / 2}=c_{21} \psi_{1}\left(0, \frac{1}{2}\right)+c_{22} \psi_{2}\left(0,-\frac{1}{2}\right) .
\end{aligned}
$$

We have

(i) $\quad w_{+}(0)=\int_{-\infty}^{\infty} l^{2}\left(x_{l}^{2}+y_{l}^{2}\right) d l+\left(\frac{1}{2}\right)^{2}\left(x_{1 / 2}^{2}+y_{1 / 2}^{2}\right)+\left(-\frac{1}{2}\right)^{2}\left(x_{-1 / 2}^{2}+y_{-1 / 2}^{2}\right)$

(ii) $\quad w_{-}(0)=\frac{1}{16}\left(\int_{-\infty}^{\infty} \frac{x_{l}^{2}+y_{l}^{2}}{l^{2}} d l+2^{2}\left(x_{1 / 2}^{2}+y_{1 / 2}^{2}\right)+(-2)^{2}\left(x_{-1 / 2}^{2}+y_{-1 / 2}^{2}\right)\right)$

(iii) $\quad \sin q(0)=\frac{-i}{8}\left(\int_{-\infty}^{\infty} \frac{\left(x_{l}^{2}-y_{l}^{2}\right)}{l} d l+2\left(x_{1 / 2}^{2}-y_{1 / 2}^{2}\right)+(-2)\left(x_{-1 / 2}^{2}-y_{-1 / 2}^{2}\right)\right)$

(iv) $)^{\prime} \quad \cos q(0)=\frac{i}{4}\left(\int_{-\infty}^{\infty} \frac{x_{l} y_{l}}{l} d l+2 x_{1 / 2} y_{1 / 2}+(-2) x_{-1 / 2} y_{-1 / 2}\right)$

(v) $\quad 0=\int_{-\infty}^{\infty} x_{l}^{2}+y_{l}^{2} d l+x_{1 / 2}^{2}+y_{1 / 2}^{2}+x_{-1 / 2}^{2}+y_{-1 / 2}^{2}$

$(\mathrm{vi})^{\prime} \quad 0=\int_{-\infty}^{\infty} l\left(x_{l}^{2}-y_{l}^{2}\right) d l+\frac{1}{2}\left(x_{1 / 2}^{2}-y_{1 / 2}^{2}\right)+\left(-\frac{1}{2}\right)\left(x_{-1 / 2}^{2}-y_{-1 / 2}^{2}\right)$

(vii) $^{\prime} \quad-i=\int_{-\infty}^{\infty} l x_{l} y_{l} d l+\frac{1}{2} x_{1 / 2} y_{1 / 2}+\left(-\frac{1}{2}\right) x_{-1 / 2} y_{-1 / 2}$.

Let

$$
\left(\begin{array}{l}
w_{1} \\
w_{2}
\end{array}\right)=\left(\begin{array}{cc}
\cos \frac{q}{2} & \sin \frac{q}{2} \\
-\sin \frac{q}{2} & \cos \frac{q}{2}
\end{array}\right)\left(\begin{array}{l}
z_{1} \\
z_{2}
\end{array}\right)
$$

then it is easy to check

$$
\left(\begin{array}{l}
w_{1} \\
w_{2}
\end{array}\right)^{\prime}=\left(\begin{array}{cc}
\frac{i}{8 k}-\frac{i k}{2} \cos q & \frac{w_{-}}{4}+\frac{i k}{2} \sin q \\
\frac{-w_{-}}{4}+\frac{i k}{2} \sin q & \frac{-i}{8 k}+\frac{i k}{2} \cos q
\end{array}\right)\left(\begin{array}{l}
w_{1} \\
w_{2}
\end{array}\right) .
$$

Set

$$
\left(\begin{array}{l}
u_{k} \\
v_{k}
\end{array}\right)=\left(\begin{array}{cc}
\cos \frac{q}{2} & \sin \frac{q}{2} \\
-\sin \frac{q}{2} & \cos \frac{q}{2}
\end{array}\right)\left(\begin{array}{l}
x_{k} \\
y_{k}
\end{array}\right) \text { for all } k .
$$


Now by substitution

$$
\begin{aligned}
& \text { (ii) } \quad w_{-}(0)=\frac{1}{16}\left(\int_{-\infty}^{\infty} \frac{u_{l}^{2}+v_{l}^{2}}{l^{2}} d l+2^{2}\left(u_{1 / 2}^{2}+v_{1 / 2}^{2}\right)+(-2)^{2}\left(u_{-1 / 2}^{2}+v_{-1 / 2}^{2}\right)\right) \\
& \text { (iii) }^{\prime \prime} \quad \sin q(0)=\frac{i}{2}\left(\int_{-\infty}^{\infty} l\left(u_{l}^{2}-v_{l}^{2}\right)+\frac{1}{2}\left(u_{1 / 2}^{2}-v_{1 / 2}^{2}\right)+\left(-\frac{1}{2}\right)\left(u_{-1 / 2}^{2}-v_{-1 / 2}^{2}\right)\right) \\
& \text { (iv) }^{\prime \prime} \cos q(0)=i\left(\int_{-\infty}^{\infty} l u_{l} v_{l} d l+\frac{1}{2} u_{1 / 2} v_{1 / 2}+\left(-\frac{1}{2}\right) u_{-1 / 2} v_{-1 / 2}\right) \\
& (\mathrm{v})^{\prime \prime} \quad 0=\int_{-\infty}^{\infty} u_{l}^{2}+v_{l}^{2} d l+u_{1 / 2}^{2}+v_{1 / 2}^{2}+u_{-1 / 2}^{2}+v_{-1 / 2}^{2} \\
& \text { (vi) }^{\prime \prime} \quad 0=\int_{-\infty}^{\infty} \frac{u_{l}^{2}-v_{l}^{2}}{l} d l+2\left(u_{1 / 2}^{2}-v_{1 / 2}^{2}\right)+(-2)\left(u_{-1 / 2}^{2}-v_{-1 / 2}^{2}\right) \\
& \text { (vii) }^{\prime \prime} \quad-4 i=\int_{-\infty}^{\infty} \frac{u_{l} v_{l}}{l}+2 u_{1 / 2} u_{1 / 2}+(-2) u_{-1 / 2} v_{-1 / 2} .
\end{aligned}
$$

It is easy to see that under the map

$$
\left(q(x, 0), \frac{\partial q}{\partial t}(x, 0)\right) \rightarrow\left(x_{k}, y_{k}, u_{k}, v_{k}\right)
$$

translation becomes

$$
\begin{aligned}
& \frac{d}{d t} x_{k}=\frac{-i k}{2} x_{k}+2 l y_{k}+\frac{a_{1}}{4 k} x_{k}-\frac{a_{2}}{2 k} y_{k} \\
& \frac{d}{d t} y_{k}=\frac{i k}{2} y_{k}-2 l x_{k}-\frac{a_{1}}{4 k} y_{k}-\frac{a_{2}}{2 k} x_{k} \\
& \frac{d}{d t} u_{k}=\frac{i}{8 k} u_{k}+2 m v_{k}-k a_{1} u_{k}-2 k a_{2} v_{k} \\
& \frac{d}{d t} v_{k}=\frac{-i}{8 k} v_{k}-2 m u_{k}+a_{1} k v_{k}-2 k a_{2} u_{k}
\end{aligned}
$$

where

$$
\begin{aligned}
l & =-\frac{1}{8}\left(\int_{-\infty}^{\infty} l^{2}\left(x_{l}^{2}+y_{l}^{2}\right) d l+\left(\frac{1}{2}\right)^{2}\left(x_{1 / 2}^{2}+y_{1 / 2}^{2}\right)+\left(-\frac{1}{2}\right)^{2}\left(x_{-1 / 2}^{2}+y_{-1 / 2}^{2}\right)\right) \\
m & =\frac{1}{8 \cdot 16}\left(\int_{-\infty}^{\infty} \frac{u^{2}+v_{l}^{2}}{l^{2}} d l+2^{2}\left(u_{1 / 2}^{2}+v_{1 / 2}^{2}\right)+(-2)^{2}\left(u_{-1 / 2}^{2}+v_{-1 / 2}^{2}\right)\right) \\
a_{1} & =-\frac{1}{8}\left(\int_{-\infty}^{\infty} \frac{x_{l} y_{l}}{l} d l+2 x_{1 / 2} y_{1 / 2}+(-2) x_{-1 / 2} y_{-1 / 2}\right) \\
a_{2} & =-\frac{1}{32}\left(\int_{-\infty}^{\infty} \frac{x_{l}^{2}-y_{l}^{2}}{l} d l+2\left(x_{1 / 2}^{2}-y_{1 / 2}^{2}\right)+(-2)\left(x_{-1 / 2}^{2}-y_{-1 / 2}^{2}\right)\right)
\end{aligned}
$$


and

$$
\begin{aligned}
0= & \int_{-\infty}^{\infty} x_{l}^{2}+y_{l}^{2} d l+x_{1 / 2}^{2}+y_{1 / 2}^{2}+x_{-1 / 2}^{2}+y_{-1 / 2}^{2}, \\
0= & \int_{-\infty}^{\infty} u_{l}^{2}+v_{l}^{2} d l+u_{1 / 2}^{2}+v_{1 / 2}^{2}+u_{-1 / 2}^{2}+v_{-1 / 2}^{2}, \\
0= & \int_{-\infty}^{\infty} l\left(x_{l}^{2}-y_{l}^{2}\right) d l+\frac{1}{2}\left(x_{1 / 2}^{2}-y_{1 / 2}^{2}\right)+\left(-\frac{1}{2}\right)\left(x_{-1 / 2}^{2}-y_{-1 / 2}^{2}\right) \\
0= & \int_{-\infty}^{\infty} \frac{u_{l}^{2}-v_{l}^{2}}{l} d l+2\left(u_{1 / 2}^{2}-v_{1 / 2}^{2}\right)+(-2)\left(u_{-1 / 2}^{2}-v_{-1 / 2}^{2}\right) \\
0= & \int_{-\infty}^{\infty}\left(\frac{x_{l} y_{l}}{4 l}-l u_{l} v_{l}\right) d l+\frac{1}{2}\left(x_{1 / 2} y_{1 / 2}-u_{1 / 2} v_{1 / 2}\right)+\left(-\frac{1}{2}\right)\left(x_{-1 / 2} y_{-1 / 2}-u_{-1 / 2} v_{-1 / 2}\right) \\
0= & \int_{-\infty}^{\infty}\left[\frac{\left(x_{l}^{2}-y_{l}^{2}\right)}{4 l}+l\left(u_{l}^{2}-v_{l}^{2}\right)\right] d l+\frac{1}{2}\left(\left(x_{1 / 2}^{2}-y_{1 / 2}^{2}\right)-\left(u_{1 / 2}^{2}-v_{1 / 2}^{2}\right)\right) \\
& +\left(-\frac{1}{2}\right)\left(\left(x_{-1 / 2}^{2}-y_{-1 / 2}^{2}\right)-\left(u_{-1 / 2}^{2}-v_{-1 / 2}^{2}\right)\right)
\end{aligned}
$$

are maintained.

We show that system (1) is an integrable constrained harmonic motion. It will be convenient to write

$$
\begin{aligned}
& \sum_{l} x_{l}^{2}+y_{l}^{2} \text { for } \int_{-\infty}^{\infty} x_{l}^{2}+y_{l}^{2} d l+x_{1 / 2}^{2}+y_{1 / 2}^{2}+x_{-1 / 2}^{2}+y_{-1 / 2}^{2}, \\
& \sum_{l} l\left(x_{l}^{2}-y_{l}^{2}\right) \text { for } \int_{-\infty}^{\infty} l\left(x_{l}^{2}-y_{l}^{2}\right) d l+\frac{1}{2}\left(x_{1 / 2}^{2}-y_{1 / 2}^{2}\right)+\left(-\frac{1}{2}\right)\left(x_{-1 / 2}^{2}-y_{-1 / 2}^{2}\right) \text { and }
\end{aligned}
$$
so on.

Let

$$
\begin{array}{ll}
\phi_{1}=\sum_{l} x_{l}^{2}+y_{l}^{2}, & \phi_{2}=\sum_{l} l\left(x_{l}^{2}-y_{l}^{2}\right) \\
\psi_{1}=\sum_{l} u_{l}^{2}+v_{l}^{2}, & \psi_{2}=\sum_{l} \frac{1}{l}\left(u_{l}^{2}-v_{l}^{2}\right) \\
\theta_{1}=\sum_{l}\left(\frac{x_{l} y_{l}}{4 l}-l u_{l} v_{l}\right), & \theta_{2}=\sum_{l} \frac{1}{4 l}\left(x_{l}^{2}-y_{l}^{2}\right)+l\left(u_{l}^{2}-v_{l}^{2}\right)
\end{array}
$$

and 
Constraining $H_{0}$ to $X=\left\{\phi_{1}=\phi_{2}=\psi_{1}=\psi_{2}=\theta_{1}=\theta_{2}=0\right\}$ we find $H=H_{0}+\lambda_{1} \phi_{1}$ $+\lambda_{2} \phi_{2}+\alpha_{1} \theta_{1}+\alpha_{2} \theta_{2}+\mu_{1} \psi_{1}+\mu_{2} \psi_{2}$ where $\alpha_{1}, \alpha_{2}, \lambda_{1}, \lambda_{2}, \mu_{1}, \mu_{2}$ are determined by $^{12}$

$$
\begin{aligned}
0= & \left\{\phi_{1}, H\right\}=\lambda_{2}\left(-8 \sum_{l} l x_{l} y_{l}\right)+\alpha_{1}\left(\frac{1}{2} \sum_{l} \frac{x_{l}^{2}-y_{l}^{2}}{l}\right)+\alpha_{2}\left(-2 \sum_{l} \frac{x_{l} y_{l}}{l}\right) \\
0= & \left\{\phi_{2}, H\right\}=\lambda_{1}\left(8 \sum_{l} l x_{l} y_{l}\right)-i \sum_{l} l^{2}\left(x_{l}^{2}+y_{l}^{2}\right) \\
0= & \left\{\theta_{1}, H\right\}=\lambda_{1}\left(-\frac{1}{2} \sum_{l} \frac{x_{l}^{2}-y_{l}^{2}}{l}\right)+\alpha_{2}\left(-\frac{1}{8} \sum_{l} \frac{x_{l}^{2}+y_{l}^{2}}{l^{2}}+2 \sum_{l} l^{2}\left(u_{l}^{2}+v_{l}^{2}\right)\right) \\
& +\mu_{1}\left(2 \sum_{l} l\left(u_{l}^{2}-v_{l}^{2}\right)\right) \\
0= & \left\{\theta_{2}, H\right\}=\lambda_{1}\left(2 \sum_{l} \frac{x_{l} y_{l}}{l}\right)+\alpha_{1}\left(\frac{1}{8} \sum_{l} \frac{x_{l}^{2}+y_{l}^{2}}{l^{2}}-2 \sum_{l} l^{2}\left(u_{l}^{2}+v_{l}^{2}\right)\right) \\
& +\mu_{1}\left(8 \sum_{l} l u_{l} v_{l}\right) \\
0= & \left\{\psi_{1}, H\right\}=\alpha_{1}\left(-2 \sum_{l} l\left(u_{l}^{2}-v_{l}^{2}\right)\right)+\alpha_{2}\left(-8 \sum_{l} l u_{l} v_{l}\right)+\mu_{2}\left(-8 \sum_{l} \frac{u_{l} v_{l}}{l}\right) \\
0= & \left\{\psi_{2}, H\right\}=\frac{i}{4} \sum_{l} \frac{u_{l}^{2}+v_{l}^{2}}{l^{2}}+\mu_{1}\left(8 \sum_{l} \frac{u_{l} v_{l}}{l}\right)
\end{aligned}
$$

on $X$ so that

$$
\begin{aligned}
& \lambda_{1}=\frac{i \sum_{l} l^{2}\left(x_{l}^{2}+y_{l}^{2}\right)}{8 \sum_{l} l x_{l} y_{l}}, \quad \lambda_{2}=0 \\
& \mu_{1}=\frac{-i \sum_{l} \frac{u_{l}^{2}+v_{l}^{2}}{l^{2}}}{32 \sum_{l} \frac{u_{l} v_{l}}{l}}, \quad \mu_{2}=0
\end{aligned}
$$

and

$$
\alpha_{1}=\gamma \sum_{l} \frac{x_{l} y_{l}}{l}, \quad \alpha_{2}=\gamma \sum_{k} \frac{x_{l}^{2}-y_{l}^{2}}{4 l}
$$

where

$$
\gamma=\frac{\left(\lambda_{1}+\mu_{1}\right)}{\sum_{l}\left[l^{2}\left(u_{l}^{2}+v_{l}^{2}\right)-\frac{1}{16}\left(\frac{x_{l}^{2}+y_{l}^{2}}{l^{2}}\right)\right]} .
$$

12 The $x_{l}$ 's and $y_{l}$ 's are canonically conjugate as are the $u_{l}$ 's and $v_{l}$ 's, while $\left\{x_{k}, u_{l}\right\}=\left\{x_{k}, v_{l}\right\}=\left\{y_{k}, u_{l}\right\}$ $=\left\{y_{k}, v_{l}\right\}=0$ 
The Hamiltonian equations of constrained motion are

$$
\begin{aligned}
& \frac{d}{d t} x_{k}=\frac{-i k}{2} x_{k}+2 \lambda_{1} y_{k}+\frac{\alpha_{1}}{4 k} x_{k}-\frac{\alpha_{2}}{2 k} y_{k} \\
& \frac{d}{d t} y_{k}=\frac{i k}{2} y_{k}-2 \lambda_{1} x_{k}-\frac{\alpha_{1}}{4 k} y_{k}-\frac{\alpha_{2}}{2 k} x_{k} \\
& \frac{d}{d t} u_{k}=\frac{i}{8 k} u_{k}+2 \mu_{1} v_{k}-\alpha_{1} k u_{k}-2 k \alpha_{2} v_{k} \\
& \frac{d}{d t} v_{k}=\frac{-i}{8 k} v_{k}-2 \mu_{1} u_{k}+\alpha_{1} k v_{k}-2 k \alpha_{2} u_{k} .
\end{aligned}
$$

Theorem 1. Let

$$
C_{k}=\sum_{l \neq k}\left(\frac{k+l}{k-l}\right)\left(x_{k} y_{l}-x_{l} y_{k}\right)^{2}+\left(\frac{k-l}{k+l}\right)\left(x_{k} x_{l}+y_{k} y_{l}\right)^{2}
$$

and

$$
D_{k}=\sum_{l \neq k}\left(\frac{k+l}{k-l}\right)\left(u_{k} v_{l}-u_{l} v_{k}\right)^{2}+\left(\frac{k-l}{k+l}\right)\left(u_{k} u_{l}+v_{k} v_{l}\right)^{2}
$$

for all $k$. Then the $C_{k}$ 's, $D_{k}$ 's Poisson commute on $X$, as well as in the ambient space, and they are conserved by the constrained motion (2).

Proof. Set $z_{k l}=x_{k} y_{l}-x_{l} y_{k}, w_{k l}=x_{k} x_{l}+y_{k} y_{l}, a_{k l}=\frac{k+l}{k-l}$ and $b_{k l}=\frac{k-l}{k+l}$ so that $C_{k}$ $=\sum_{l \neq k} a_{k l} z_{k l}^{2}+b_{k l} w_{k l}^{2}$ for all $k$. Now

$$
\begin{aligned}
\left\{C_{k}, C_{i}\right\}= & \left\{\sum_{l \neq k} a_{k l} z_{k l}^{2}+b_{k l} w_{k l}^{2}, \sum_{j \neq i} a_{i j} z_{i j}^{2}+b_{i j} w_{i j}^{2}\right\} \\
= & 4 \sum_{l \neq k, j \neq i} a_{k l} a_{i j} z_{k l} z_{i j}\left\{z_{k l}, z_{i j}\right\} \\
& +4 \sum_{l \neq k, j \neq i} a_{k l} b_{i j} z_{k l} w_{i j}\left\{z_{k l}, w_{i j}\right\} \\
& +4 \sum_{l \neq k, j \neq i} a_{i j} b_{k l} z_{i j} w_{k l}\left\{w_{k l}, z_{i j}\right\} \\
& +4 \sum_{l \neq k, j \neq i} b_{k l} b_{i j} w_{k l} w_{i j}\left\{w_{k l}, w_{i j}\right\} .
\end{aligned}
$$

By direct calculation

$$
\begin{aligned}
& 4 \sum_{l \neq k, j \neq i} a_{k l} a_{i j} z_{k l} z_{i j}\left\{z_{k l}, z_{i j}\right\}=4 z_{i k} \sum_{l \neq i, k}\left(a_{k l} a_{i k}-a_{k l} a_{i l}+a_{k i} a_{i l}\right) z_{k l} z_{i l} \\
& 4 \sum_{l \neq k, j \neq i} a_{k l} b_{i j} z_{k l} w_{i j}\left\{z_{k l}, w_{i j}\right\} \\
& \quad=4 w_{i k} \sum_{l \neq k}\left(a_{k l} b_{i k}-a_{k l} b_{i l}\right) z_{k l} w_{i l}-4 z_{k i} \sum_{l} a_{k i} b_{i l} w_{i l} w_{k l}, \\
& 4 \sum_{l \neq k, j \neq i} a_{i j} b_{k l} z_{i j} w_{k l}\left\{w_{k l}, z_{i j}\right\} \\
& \quad=4 w_{k i} \sum_{l \neq i}\left(a_{i l} b_{k l}-a_{i l} b_{k i}\right) z_{i l} w_{k l}+4 z_{i k} \sum_{l} a_{i k} b_{k l} w_{k l} w_{i l}
\end{aligned}
$$


and

$$
\begin{aligned}
& 4 \sum_{l \neq k, j \neq i} b_{k l} b_{i j} w_{k l} w_{i j}\left\{w_{k l}, w_{i j}\right\} \\
& \quad=4 w_{i k} \sum_{l} b_{k l} b_{i k} w_{k l} z_{l i}+b_{k i} b_{i l} w_{i l} z_{k l}+4 z_{k i} \sum_{l} b_{k l} b_{i l} w_{k l} w_{i l} .
\end{aligned}
$$

We have

$$
\begin{aligned}
\left\{C_{k}, C_{i}\right\}= & 4 z_{i k} \sum_{l \neq i, k}\left(a_{k l} a_{i k}-a_{k l} a_{i l}+a_{k i} a_{i l}\right) z_{k l} z_{i l} \\
& +4 z_{i k} \sum_{l}\left(a_{i k} b_{k l}-a_{i k} b_{i l}-b_{k l} b_{i l}\right) w_{i l} w_{k l} \\
& +4 w_{i k} \sum_{l \neq k}\left(a_{k l} b_{i k}-a_{k l} b_{i l}+b_{k i} b_{i l}\right) w_{i l} z_{k l} \\
& +4 w_{i k} \sum_{l \neq i}\left(a_{i l} b_{k l}-a_{i l} b_{k i}-b_{k l} b_{i k}\right) w_{k l} z_{i l} .
\end{aligned}
$$

Also,

$$
\begin{aligned}
& a_{k l} a_{i k}-a_{k l} a_{i l}+a_{k i} a_{i l}=-1, \\
& a_{i k} b_{k l}-a_{i k} b_{i l}-b_{k l} b_{i l}=-1, \\
& a_{k l} b_{i k}-a_{k l} b_{i l}+b_{k i} b_{i l}=-1, \\
& a_{i l} b_{k l}-a_{i l} b_{k i}-b_{k l} b_{i k}=1,
\end{aligned}
$$

so that

$$
\left\{C_{k}, C_{i}\right\}=-4 z_{i k} \sum_{l}\left(z_{k l} z_{i l}+w_{i l} w_{k l}\right)+4 w_{i k} \sum_{l}\left(z_{i l} w_{k l}-z_{k l} w_{i l}\right) .
$$

However,

$$
z_{k l} z_{i l}+w_{i l} w_{k l}=w_{i k}\left(x_{l}^{2}+y_{l}^{2}\right)
$$

and

$$
z_{i l} w_{k l}-z_{k l} w_{i l}=z_{i k}\left(x_{l}^{2}+y_{l}^{2}\right) .
$$

Thus

$$
\left\{C_{k}, C_{i}\right\}=-4 z_{i k} w_{i k} \sum_{l}\left(x_{l}^{2}+y_{l}^{2}\right)+4 z_{i k} w_{i k} \sum_{l}\left(x_{l}^{2}+y_{l}^{2}\right)=0,
$$

i.e., the $C_{k}$ 's Poisson commute in the ambient space.

Let $\chi_{1}=\phi_{2}, \chi_{2}=\theta_{1}, \chi_{3}=\theta_{2}, \chi_{4}=\psi_{2}, \chi_{5}=\phi_{1}, \chi_{6}=\psi_{1}$, and $d=\left(\left\{\chi_{i}, \chi_{j}\right\}\right)$. Then

$$
\begin{aligned}
\left\{C_{k}, C_{l}\right\}_{X} & =\left\{C_{k}, C_{l}\right\}-\sum_{i, j=1}^{6}\left\{C_{k}, \chi_{i}\right\} d_{i j}^{-1}\left\{\chi_{j}, C_{l}\right\} \\
& =\sum_{i, j=1}^{3}\left\{C_{k}, \chi_{i}\right\} d_{i j}^{-1}\left\{\chi_{j}, C_{l}\right\} \\
& =\sum_{1 \leqq i<j \leqq 3} d_{i j}^{-1}\left(\left\{C_{k}, \chi_{i}\right\}\left\{\chi_{j}, C_{l}\right\}-\left\{C_{k}, \chi_{j}\right\}\left\{\chi_{i}, C_{l}\right\}\right)
\end{aligned}
$$


since $\left\{C_{k}, \phi_{1}\right\}=\left\{C_{k}, \psi_{1}\right\}=\left\{C_{k}, \psi_{2}\right\}=0$ for all $k$. By direct calculation

$$
\begin{aligned}
& \left\{\phi_{2}, C_{k}\right\}=\left(x_{k}^{2}+y_{k}^{2}\right)\left\{\phi_{1}, \phi_{2}\right\}+8 k x_{k} y_{k} \phi_{1}, \\
& \left\{\theta_{1}, C_{k}\right\}=-\left(x_{k}^{2}+y_{k}^{2}\right)\left\{\phi_{1}, \theta_{1}\right\}+\left(\frac{x_{k}^{2}-y_{k}^{2}}{2 k}\right) \phi_{1},
\end{aligned}
$$

and

$$
\left\{\theta_{2}, C_{k}\right\}=-\left(x_{k}^{2}+y_{k}^{2}\right)\left\{\phi_{1}, \theta_{2}\right\}-2 \frac{y_{k} y_{k}}{k} \phi_{1}
$$

so that

$$
\begin{aligned}
\left\{C_{k}, C_{l}\right\}_{X}= & d_{12}^{-1}\left(\left\{C_{k}, \chi_{1}\right\}\left\{\chi_{2}, C_{l}\right\}-\left\{C_{k}, \chi_{2}\right\}\left\{\chi_{1}, C_{l}\right\}\right) \\
& +d_{23}^{-1}\left(\left\{C_{k}, \chi_{2}\right\}\left\{\chi_{3}, C_{l}\right\}-\left\{C_{k}, \chi_{3}\right\}\left\{\chi_{2}, C_{l}\right\}\right) \\
& +d_{13}^{-1}\left(\left\{C_{k}, \chi_{1}\right\}\left\{\chi_{3}, C_{l}\right\}-\left\{C_{k}, \chi_{3}\right\}\left\{\chi_{1}, C_{l}\right\}\right) \\
= & d_{12}^{-1}\left(x_{k}^{2}+y_{k}^{2}\right)\left(x_{l}^{2}+y_{l}^{2}\right)\left(\left\{\phi_{1}, \phi_{2}\right\}\left\{\phi_{1}, \theta_{1}\right\}-\left\{\phi_{1}, \theta_{1}\right\}\left\{\phi_{1}, \phi_{2}\right\}\right) \\
& +d_{23}^{-1}\left(x_{k}^{2}+y_{k}^{2}\right)\left(x_{l}^{2}+y_{l}^{2}\right)\left(\left\{\phi_{1}, \theta_{2}\right\}\left\{\phi_{1}, \theta_{1}\right\}-\left\{\phi_{1}, \theta_{1}\right\}\left\{\phi_{1}, \theta_{2}\right\}\right) \\
& +d_{13}^{-1}\left(x_{k}^{2}+y_{k}^{2}\right)\left(x_{l}^{2}+y_{l}^{2}\right)\left(\left\{\phi_{1}, \theta_{2}\right\}\left\{\phi_{1}, \phi_{2}\right\}-\left\{\phi_{1}, \theta_{2}\right\}\left\{\phi_{1}, \phi_{2}\right\}\right) \\
& + \text { terms proportional to } \phi_{1} \\
= & 0 \text { on } X .
\end{aligned}
$$

i.e., the $C_{k}$ 's Poisson commute on $X$.

Also

$$
\begin{aligned}
\frac{d}{d t} C_{k}= & 2 \sum_{l \neq k} a_{k l} z_{k l}\left(\frac{i}{2}(l-k)\left(x_{k} y_{l}+x_{l} y_{k}\right)+\frac{\alpha_{1}}{4}\left(\frac{1}{k}-\frac{1}{l}\right)\left(x_{k} y_{l}+x_{l} y_{k}\right)\right. \\
& \left.+\frac{\alpha_{2}}{2}\left(\frac{1}{l}-\frac{1}{k}\right)\left(y_{k} y_{l}-x_{k} x_{l}\right)\right) \\
& +2 \sum_{l} b_{k l} w_{k l}\left(\frac{i}{2}(k+l)\left(y_{k} y_{l}-x_{k} y_{l}\right)+\frac{\alpha_{1}}{4}\left(\frac{1}{k}+\frac{1}{l}\right)\left(x_{k} x_{l}-y_{k} y_{l}\right)\right. \\
& \left.-\frac{\alpha_{2}}{2}\left(\frac{1}{k}+\frac{1}{l}\right)\left(x_{k} y_{l}+x_{l} y_{k}\right)\right) \\
= & -i k x_{k}^{2} \sum_{l}\left(x_{l}^{2}+y_{l}^{2}\right)+i k y_{k}^{2} \sum_{l}\left(x_{l}^{2}+y_{l}^{2}\right) \\
& +i x_{k}^{2} \sum_{l} l\left(x_{l}^{2}-y_{l}^{2}\right)+i y_{k}^{2} \sum_{l} l\left(x_{l}^{2}-y_{l}^{2}\right) \\
& \left.+\frac{\alpha_{1}}{2 k}\left(y_{k}^{2}-x_{k}^{2}\right) \sum\left(x_{l}^{2}+y_{l}^{2}\right)+\frac{\alpha_{1}}{2}\left(x_{k}^{2}+y_{k}^{2}\right) \sum_{l} \frac{x_{l}^{2}-y_{l}^{2}}{2}\right\} \mathrm{II} \\
& \left.+\frac{2 \alpha_{2}}{k} x_{k} y_{k} \sum_{l}\left(x_{l}^{2}+y_{l}^{2}\right)-2 \alpha_{2}\left(x_{k}^{2}+y_{k}^{2}\right) \sum_{l} \frac{x_{l} y_{l}}{l}\right\} \mathrm{III} .
\end{aligned}
$$


On $X$

$$
\begin{aligned}
& I=0 \\
& I I=2 \frac{\alpha_{1} \alpha_{2}}{\gamma}\left(x_{k}^{2}+y_{k}^{2}\right)
\end{aligned}
$$

and

$$
\mathrm{III}=-2 \frac{\alpha_{1} \alpha_{2}}{\gamma}\left(x_{k}^{2}+y_{k}^{2}\right)
$$

Thus $\frac{d}{d t} C_{k}=0$ on $X$, i.e., the $C_{k}$ 's are conserved by the constrained motion (2).

The remaining assertions are verified by similar calculations. The proof is finished.

System (1) is obtained from (2) by imposing further constraints and symmetries.

Lemma 2. $\sum_{l} l x_{l} y_{l}, \sum_{l} \frac{u_{l} v_{l}}{l}$ and $\left(\sum_{l} \frac{x_{l} y_{l}}{l}\right)^{2}+\left(\frac{1}{2} \sum_{l} \frac{x_{l}^{2}-y_{l}^{2}}{l}\right)^{2}$ are conserved by the constrained motion (2).

Proof. Direct calculation.

Now

$$
\lambda_{1}=-\frac{1}{8} \sum_{l} l^{2}\left(x_{l}^{2}+y_{l}^{2}\right)=l
$$

and

$$
\mu_{1}=\frac{1}{8 \cdot 16} \sum_{l} \frac{u_{l}^{2}+v_{l}^{2}}{l^{2}}=m
$$

on the orbits which satisfy

$$
\begin{aligned}
& \sum_{l} l x_{l} y_{l}=-i \\
& \sum_{i} \frac{u_{l} v_{l}}{l}=-4 i
\end{aligned}
$$

Also, we can define

$$
\begin{aligned}
& \cos q=\frac{i}{4} \sum_{l} \frac{x_{l} y_{l}}{l}, \\
& \sin q=-\frac{i}{8} \sum_{l} \frac{x_{l}^{2}-y_{l}^{2}}{l}
\end{aligned}
$$


when

$$
\left(\sum_{l} \frac{x_{l} y_{l}}{l}\right)^{2}+\left(\frac{1}{2} \sum_{l} \frac{x_{l}^{2}-y_{l}^{2}}{l}\right)^{2}=-16
$$

with the result that system (2) becomes

$$
\begin{aligned}
\frac{d x_{k}}{d t} & =-\frac{i k}{2} x_{k}+2 \lambda_{1} y_{k}+\frac{\gamma}{i k}\left(x_{k} \cos q+y_{k} \sin q\right) \\
\frac{d y_{k}}{d t} & =\frac{i k}{2} y_{k}-2 \lambda_{1} x_{k}-\frac{\gamma}{i k}\left(y_{k} \cos q-x_{k} \sin q\right) \\
\frac{d u_{k}}{d t} & =-\frac{i}{8 k} u_{k}-\frac{4 \gamma k}{i}\left(u_{k} \cos q-v_{k} \sin q\right)+2 \mu_{1} v_{k} \\
\frac{d v_{k}}{d t} & =-\frac{i}{8 k} v_{k}+\frac{4 \gamma k}{i}\left(v_{k} \cos q+u_{k} \sin q\right)-2 \mu_{1} u_{k} .
\end{aligned}
$$

By direct calculation, it is easy to see that

$$
\left(\begin{array}{l}
u_{k} \\
v_{k}
\end{array}\right)=\left(\begin{array}{cc}
\cos q / 2 & \sin q / 2 \\
-\sin q / 2 & \cos q / 2
\end{array}\right)\left(\begin{array}{l}
x_{k} \\
y_{k}
\end{array}\right)
$$

is a solution of $(2 * *)$ provided

$$
\gamma \equiv-\frac{1}{8} \quad \text { and } \quad \frac{\dot{q}}{4}=\mu_{1}-\lambda_{1}
$$

Finally, one can check that any solution with

$$
\left(\begin{array}{l}
u_{k}(0) \\
v_{k}(0)
\end{array}\right)=\left(\begin{array}{cc}
\cos \frac{q(0)}{2} & \sin \frac{q(0)}{2} \\
-\sin \frac{q(0)}{2} & \cos \frac{q(0)}{2}
\end{array}\right)\left(\begin{array}{l}
x_{k}(0) \\
y_{k}(0)
\end{array}\right)
$$

maintains this symmetry for all time, and that

$$
\gamma(t) \equiv-\frac{1}{8}, \quad \frac{1}{4} \dot{q}=\mu_{1}-\lambda_{1} .
$$

Let $\left(\begin{array}{l}x_{k} \\ y_{k}\end{array}\right),\left(\begin{array}{l}u_{k} \\ v_{k}\end{array}\right)$ be a solution of $\left(2^{*}\right),\left(2^{* *}\right)$, satisfying $(*)$ and set $2 \lambda_{1}=-(\dot{q}+p) / 4$. Then $2 \mu_{1}=(\dot{q}-p) / 4$ and $(2 *),\left(2^{* *}\right)$ are identical to (1).

The Sine-Gordon equation is treated in exactly the same way. If $q(x, 0)$, $\frac{\partial}{\partial t} q(x, 0)$ are given and

$$
\frac{\partial^{2} q}{d t^{2}}-\frac{\partial^{2} q}{\partial x^{2}}+\sin q=0
$$




$$
\begin{aligned}
& \frac{d}{d t}\left(\begin{array}{l}
x_{k} \\
y_{k}
\end{array}\right)=\left(\begin{array}{cc}
-\frac{i k}{2}-\frac{i}{8 k} \cos q & -\frac{w_{+}}{4}-\frac{i}{8 k} \sin q \\
\frac{w_{+}}{4}-\frac{i}{8 k} \sin q & \frac{i k}{2}+\frac{i}{8 k} \cos q
\end{array}\right)\left(\begin{array}{l}
x_{k} \\
y_{k}
\end{array}\right) \\
& -\frac{d}{d t}\left(\begin{array}{l}
u_{k} \\
v_{k}
\end{array}\right)=\left(\begin{array}{cc}
\frac{i}{8 k}+\frac{i k}{2} \cos 2 & \frac{w_{-}}{4}-\frac{i k}{2} \sin q \\
-\frac{w_{-}}{4}-\frac{i k}{2} \sin q & -\frac{i}{8 k}-\frac{i k}{2} \cos q
\end{array}\right)\left(\begin{array}{l}
u_{k} \\
v_{k}
\end{array}\right) .
\end{aligned}
$$

System (3) is the flow generated by the Hamiltonian

$$
\frac{1}{16}\left[\sum_{k}\left(\frac{1}{16 k^{2}}-k^{2}\right) C_{k}-\left(k^{2}-\frac{1}{16 k^{2}}\right) D_{k}\right]
$$

on $X$

$\sum_{l} l x_{l} y_{l}=-i, \quad \sum_{i} \frac{u_{l} v_{l}}{l}=-4 i$

$$
\left(\sum_{l} \frac{x_{l} y_{l}}{l}\right)^{2}+\left(\frac{1}{2} \sum_{l} \frac{x_{l}^{2}-y_{l}^{2}}{l}\right)^{2}=-16,
$$

$\gamma=-1 / 8$ and

Also system (2) is the flow generated by

$$
\frac{1}{16}\left[\sum_{k}\left(\frac{1}{16 k^{2}}-k^{2}\right) C_{k}+\left(k^{2}-\frac{1}{16 k^{2}}\right) D_{k}\right] \text {. }
$$

\section{The Toda Differential Equations}

The Toda differential equations

$$
\ddot{x}_{i}=e^{x_{i-1}-x_{i}}-e^{x_{i}-x_{i+1}}, \quad i=1, \ldots, n,
$$

describe the motion of particles interacting with exponential forces. The particles move on the line when $x_{0}=-\infty, x_{n+1}=\infty$ and on the circle when $x_{i+n}=x_{i}$, $i=1, \ldots, n$. We will show that in both cases the Toda system can be mapped to a system of particles constrained to an algebraic variety. Again, the constrained motion is integrable.

The first step is to make the change of variables due to Flaschka

$$
a_{i}=\frac{1}{2} e^{\left(x_{i}-x_{i}+1\right) / 2}, \quad b_{i}=-\frac{1}{2} \dot{x}_{i}, \quad i=1, \ldots, n .
$$

For particles on the line $a_{0}=a_{n}=0$ and for particles on the circle $a_{i+n}=a_{i}, b_{i+n}=b_{i}$, $i=1, \ldots, n$.

\section{(i) Particles on the Line [6]}

Let $\left(a_{1}, \ldots, a_{n-1} ; b_{1}, \ldots, b_{n}\right) \in\left(R^{+}\right)^{n-1} \oplus R^{n}$ and let $L$ be the Dirichlet difference operator defined by

$$
L f=\left(a_{l-1} f(l-1)+b_{l} f(l)+a_{l} f(l+1), l=1, \ldots, n\right),
$$

where $a_{0}=a_{n}=0$. 
Lemma 1. The spectrum of $L$ is simple.

Proof. First, if $L f=\lambda f$ and $f(1)=0$ then $f \equiv 0$ since $f(2)=\left(\lambda-\frac{b_{1}}{a_{1}}\right) f(1)=0$ and so on. Now suppose $\lambda$ is an eigenvalue with both $L f=\lambda f, L g=\lambda g$. Then $L\left(f-\frac{f(1)}{g(1)} g\right)=\lambda\left(f-\frac{f(1)}{g(1)} g\right)$ and $\left(f-\frac{f(1)}{g(1)} g\right)(1)=0$ so that $f \equiv \frac{f(1)}{g(1)} g$.

Now let $\lambda_{1}<\ldots<\lambda_{n}$ be the eigenvalues of $L$ and $f_{1}, \ldots, f_{n}$ the corresponding normalized eigenfunctions with $f_{i}(1)>0, i=1, \ldots, n$. Set $U=\left(f_{i}(j)\right)$. We have

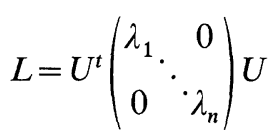

from which it immediately follows that

$$
b_{l}=\sum_{i=1}^{n} \lambda_{i} f_{i}^{2}(l), \quad l=1, \ldots, n,
$$

and

$$
a_{l}=\sum_{i=1}^{n} \lambda_{i} f_{i}(l) f_{i}(l+1), \quad l=1, \ldots, n-1
$$

Theorem 1. The map

$$
\begin{aligned}
& \left(R^{+}\right)^{n-1} \oplus R^{n} \ni\left(a_{1}, \ldots, a_{n-1} ; b_{1}, \ldots, b_{n}\right) \\
& \quad \longrightarrow\left(\lambda_{1}, \ldots, \lambda_{n} ; f_{1}(1), \ldots, f_{n}(1)\right) \in R^{n} \oplus\left(R^{+}\right)^{n}
\end{aligned}
$$

is one to one and onto

$$
\left\{\left(\lambda_{1}, \ldots, \lambda_{n} ; f_{1}(1), \ldots, f_{n}(1)\right) \in R^{n} \oplus\left(R^{+}\right)^{n}: \lambda_{1}<\ldots<\lambda_{n}, \sum_{i=1}^{n} f_{i}^{2}(1)=1\right\} .
$$

Proof. We can recover $b_{1}=\sum_{i=1}^{n} \lambda_{i} f_{i}^{2}(1)$ and hence $a_{1} f_{l}(2)=\left(\lambda_{l}-b_{1}\right) f_{l}(1), l=1, \ldots, n$ from the data $\left(\lambda_{1}, \ldots, \lambda_{n} ; f_{1}(1), \ldots, f_{n}(1)\right)$. Since $a_{1}^{2}=\sum_{i=1}^{n} \lambda_{i} f_{i}(1)\left(a_{1} f_{i}(2)\right)$ we know $a_{1}>0$ and $f_{l}(2)=\frac{\left(a_{1} f_{l}(2)\right)}{a_{1}}, l=1, \ldots, n$. Now we can recover $b_{2}=\sum_{i=1}^{n} \lambda_{i} f_{i}^{2}(2)$ and hence $a_{2} f_{l}(3)=\left(\lambda_{l}-b_{2}\right) f_{l}(2)-a_{1} f_{l}(1), l=1, \ldots, n$. Continuing in this way $L$ can be reconstructed from $\left(\lambda_{1}, \ldots, \lambda_{n} ; \mathrm{f}_{1}(1), \ldots, f_{n}(1)\right)$ and the map is one to one.

To see that the map is onto let $\lambda_{1}<\ldots<\lambda_{n}$ and $\sum_{i=1}^{n} f_{i}^{2}(1)=1$ with $f_{l}(1)>0$, $l=1, \ldots, n$. Set $b_{1}=\sum_{i=1}^{n} \lambda_{i} f_{i}^{2}(1)$. We have $\sum_{i=1}^{n}\left(\lambda_{i} f_{i}(1)-b_{1} f_{i}(1)\right)^{2}>0$; otherwise $\lambda_{l} f_{l}(1)$ $=b_{1} f_{l}(1)$ for all $1 \leqq l \leqq n$ which is impossible. Now set

$$
a_{1}=\sqrt{\sum_{i=1}^{n}\left(\lambda_{i} f_{i}(1)-b_{1} f_{i}(1)\right)^{2}}>0
$$


and

$$
f_{l}(2)=\frac{\left(\lambda_{l}-b_{1}\right) f_{l}(1)}{a_{1}}, \quad l=1, \ldots, n .
$$

Observe that

$$
\begin{aligned}
& \sum_{i=1}^{n} f_{i}(1) f_{i}(2)=\frac{1}{a_{1}} \sum_{i=1}^{n} f_{i}(1)\left(\lambda_{i}-b_{1}\right) f_{i}(1) \\
& =\frac{1}{a_{1}}\left(\sum_{i=1}^{n} \lambda_{i} f_{i}^{2}(1)-b_{1} \sum_{i=1}^{n} f_{i}^{2}(1)\right) \\
& =0 \text {, } \\
& a_{1}=\frac{1}{a_{1}} \sum_{i=1}^{n}\left(\lambda_{i} f_{i}(1)-b_{1} f_{i}(2)\right)^{2} \\
& =\sum_{i=1}^{n} f_{i}(2)\left(\lambda_{i}-b_{1}\right) f_{i}(1) \\
& =\sum_{i=1}^{n} \lambda_{i} f_{i}(1) f_{i}(2),
\end{aligned}
$$

and

$$
\sum_{i=1}^{n} f_{i}^{2}(2)=\frac{1}{a_{1}} \sum_{i=1}^{n} f_{i}(2)\left(\lambda_{i}-b_{1}\right) f_{i}(1)=1 .
$$

Set $b_{2}=\sum_{i=1}^{n} \lambda_{i} f_{i}^{2}(2)$. If $n>2$,

$$
\sum_{i=1}^{n}\left(\lambda_{i} f_{i}(2)-b_{2} f_{i}(2)-a_{1} f_{i}(1)\right)^{2}>0
$$

Otherwise, $b_{1} f_{l}(1)+a_{1} f_{l}(2)=\lambda_{l} f_{l}(1), a_{1} f_{l}(1)+b_{2} f_{l}(2)=\lambda_{2} f_{l}(2)$ for all $1 \leqq l \leqq n$ and it follows that the matrix $\left(\begin{array}{ll}b_{1} & a_{1} \\ a_{1} & b_{2}\end{array}\right)$ has more than two distinct eigenvalues which is impossible. Now set

$$
a_{2}=\sqrt{\sum_{i=1}^{n}\left(\lambda_{i} f_{i}(2)-b_{2} f_{i}(2)-a_{1} f_{i}(1)\right)^{2}}>0,
$$

and

$$
f_{l}(3)=\frac{\left(\lambda_{l}-b_{2}\right) f_{2}(2)-a_{1} f_{l}(1)}{a_{2}}, \quad l=1, \ldots, n .
$$

As before $\sum_{i=1}^{n} f_{i}(1) f_{i}(3)=0, \quad \sum_{i=1}^{n} f_{i}(2) f_{i}(3)=0$,

$$
\begin{aligned}
a_{2} & =\sum_{i=1}^{n} f_{i}(3)\left(\lambda_{i} f_{i}(2)-b_{2} f_{i}(2)-a_{1} f_{i}(1)\right) \\
& =\sum_{i=1}^{n} \lambda_{i} f_{i}(2) f_{i}(3)
\end{aligned}
$$


and

$$
\begin{aligned}
\sum_{i=1}^{n} f_{i}^{2}(3) & =\frac{1}{a_{2}} \sum_{i=1}^{n} f_{i}(3)\left(\lambda_{i} f_{i}(2)-b_{2} f_{i}(2)-a_{1} f_{i}(1)\right) \\
& =\frac{1}{a_{2}} \sum_{i=1}^{n} \lambda_{i} f_{i}(2) f_{i}(3) \\
& =1 .
\end{aligned}
$$

Repeating this construction $n-1$ times we obtain $\left(a_{1}, \ldots, a_{n-1} ; b_{1}, \ldots, b_{n}\right)$ $\in\left(R^{+}\right)^{n} \oplus R^{n}$ and a unit perpendicular frame $\left(f_{l}(k)\right), 1 \leqq k, l \leqq n$, satisfying

$$
\begin{aligned}
& b_{1} f_{l}(1)+a_{1} f_{l}(2)=\lambda_{l} f_{l}(1), \quad l=1, \ldots, n . \\
& a_{k-1} f_{l}(k-1)+b_{k} f_{l}(k)+a_{k} f_{l}(k+1)=\lambda_{l} f_{l}(k), \quad l=1, \ldots, n,
\end{aligned}
$$

and

$$
\begin{aligned}
& a_{l}=\sum_{i=1}^{n} \lambda_{i} f_{i}(l) f_{i}(l+1), \quad l=1, \ldots, n-1, k=2, \ldots, n-1, \\
& b_{l}=\sum_{i=1}^{n} \lambda_{i} f_{i}^{2}(l), \quad l=1, \ldots n, .
\end{aligned}
$$

It only remains to show that

$$
a_{n-1} f_{l}(n-1)+b_{n} f_{l}(n)=\lambda_{l} f_{l}(n), \quad l=1, \ldots, n .
$$

But for $k=1, \ldots, n-2$,

$$
\begin{aligned}
& \sum_{i=1}^{n} f_{i}(k)\left(a_{n-1} f_{i}(n-1)+b_{n} f_{i}(n)-\lambda_{i} f_{i}(n)\right) \\
& \quad=-\sum_{i=1}^{n} \lambda_{i} f_{i}(k) f_{i}(n) \\
& \quad=-\sum_{i=1}^{n}\left(a_{k-1} f_{i}(k-1)+b_{k} f_{i}(k)+a_{k} f_{i}(k+1)\right) f_{i}(n) \\
& \quad=0 .
\end{aligned}
$$

Also,

$$
\begin{aligned}
& \sum_{i=1}^{n} f_{i}(n-1)\left(a_{n-1} f_{i}(n-1)+b_{n} f_{i}(n)-\lambda_{i} f_{i}(n)\right) \\
& \quad=a_{n-1} \sum_{i=1}^{n} f_{i}^{2}(n-1)-\sum_{i=1}^{n} \lambda_{i} f_{i}(n-1) f_{i}(n) \\
& \quad=a_{n-1}-\sum_{i=1}^{n} \lambda_{i} f_{i}(n-1) f_{i}(n) \\
& \quad=0
\end{aligned}
$$


and

$$
\begin{aligned}
& \sum_{i=1}^{n} f_{i}(n)\left(a_{n-1} f_{i}(n-1)+b_{n} f_{i}(n)-\lambda_{i} f_{i}(n)\right) \\
& \quad=b_{n} \sum_{i=1}^{n} f_{i}^{2}(n)-\sum_{i=1}^{n} \lambda_{i} f_{i}^{2}(n) \\
& \quad=b_{n}-\sum_{i=1}^{n} \lambda_{i} f_{i}^{2}(n)=0
\end{aligned}
$$

The proof is finished.

Theorem 2. The system of differential equations

$$
\begin{aligned}
& \frac{d}{d t} a_{l}=a_{l}\left(b_{l+1}-b_{l}\right), \\
& \frac{d}{d t} b_{l}=2\left(a_{l}^{2}-a_{l-1}^{2}\right),
\end{aligned}
$$

with $a_{0}=a_{n}=0$ is mapped to

$$
\begin{aligned}
\frac{d}{d t} \lambda_{l} & =0 \\
\frac{d}{d t} f_{l}(1) & =\left(\lambda_{l}-\sum_{i=1}^{n} \lambda_{i} f_{i}^{2}(1)\right) f_{l}(1) .
\end{aligned}
$$$$
l=1, \ldots, n
$$

Proof. System (1) can be written as $\frac{d}{d t} L=B L-L B$ where

$$
L=\left(\begin{array}{ccc}
b_{1} a_{1} & 0 \\
a_{1} b_{2} & \ddots \\
& \ddots b_{n-1} a_{n-1} \\
0 & & a_{n-1} b_{n}
\end{array}\right)
$$

and

$$
B=\left(\begin{array}{ccccc}
0 & a_{1} & & & 0 \\
-a_{1} & 0 & \ddots & & \\
& & & 0 & a_{n-1} \\
0 & & & -a_{n-1} & 0
\end{array}\right) .
$$

Let $\frac{d}{d t} V=-V B, V(0)=I$. Then $\frac{d}{d t} V V^{t}=0$ and $\frac{d}{d t} V L V^{t}=0$, so that $V L V^{-1}=L(0)$ giving $\frac{d}{d t} \lambda_{l}=0, l=1, \ldots, n$.

Now differentiate the equation $L f_{l}=\lambda_{l} f_{l}$ to obtain $L\left(\frac{d}{d t} f_{l}-B f_{l}\right)$ 
$=\lambda_{l}\left(\frac{d}{d t} f_{l}-B f_{l}\right)$ and hence $\frac{d}{d t} f_{l}-B f_{l}=c f_{l}$ since $\lambda_{l}$ is simple. However $c=\left(\frac{d}{d t} f_{l}, f_{l}\right)$ $-\left(B f_{l}, f_{l}\right)=0$ because $\left(f_{l}, f_{l}\right)=1$ and $B$ is skew. We have $\frac{d}{d t} f_{l}=B f_{l}$. In particular

$$
\begin{aligned}
\frac{d}{d t} f_{l}(1) & =a_{1} f_{l}(2)=\left(\lambda_{l}-b_{1}\right) f_{l}(1) \\
& =\left(\lambda_{l}-\sum_{i=1}^{n} \lambda_{i} f_{i}^{2}(1)\right) f_{l}(1)
\end{aligned}
$$

The proof is finished.

The form $\omega_{+}=\sum_{i=1}^{n} \frac{d x_{i} \wedge d y_{i}}{x_{i}}$ defines a symplectic structure on $\left(R^{+}\right)^{n} \oplus R^{n}$ with Poisson bracket $\{,\}_{+}$, satisfying $\left\{x_{k}, x_{l}\right\}_{+}=\left\{y_{k}, y_{l}\right\}_{+}=0$ and $\left\{x_{k}, y_{l}\right\}_{+}=\delta_{k l} x_{k}$ for all $1 \leqq k, l \leqq n$. Let $H_{0}=\frac{1}{2} \sum_{i=1}^{n} y_{i}^{2}$. Then the corresponding Hamiltonian equations are

$$
\begin{array}{ll}
\frac{d}{d t} x_{l}=\left\{x_{l}, H_{0}\right\}_{+}=y_{l} x_{l}, & \\
\frac{d}{d t} y_{l} & =\left\{y_{l}, H_{0}\right\}_{+}=0,
\end{array} \quad l=1, \ldots, n .
$$

Let $\phi_{1}=\sum_{i=1}^{n} x_{i}^{2}-1, \phi_{2}=\sum_{i=1}^{n} y_{i}-c$. We have $\left\{\phi_{1}, \phi_{2}\right\}_{+}=2\left(\phi_{1}+1\right),\left\{\phi_{1}, \mathrm{H}_{0}\right\}_{+}$ $=2 \sum_{i=1}^{n} x_{i}^{2} y_{i}$ and $\left\{\phi_{2}, H_{0}\right\}_{+}=0$. Constraining $H_{0}$ to $X=\left\{\phi_{1}=\phi_{2}=0\right\}$ we find $H=H_{0}+\alpha_{1} \phi_{1}+\alpha_{2} \phi_{2}$ where $\alpha_{1}, \alpha_{2}$ are determined by

$$
\begin{aligned}
0 & =\left\{\phi_{1}, H\right\}_{+}=2 \sum_{i=1}^{n} x_{i}^{2} y_{i}+\alpha_{2} 2\left(\phi_{1}+1\right) \\
& =2 \sum_{i=1}^{n} x_{i}^{2} y_{i}+2 \alpha_{2}, \\
0 & =\left\{\phi_{2}, H\right\}_{+}=-\alpha_{1} 2\left(\phi_{1}+1\right)=-2 \alpha_{1},
\end{aligned}
$$

on $X$ so that $\alpha_{1}=0$ and $\alpha_{2}=-\sum_{i=1}^{n} x_{i}^{2} y_{i}$. Finally, the equations of constrained motion are

$$
\begin{aligned}
& \frac{d}{d t} x_{l}=\left\{x_{l}, H\right\}_{+}=\left(y_{l}-\sum_{i=1}^{n} x_{i}^{2} y_{i}\right) x_{l}, \quad l=1, \ldots, n . \\
& \frac{d}{d t} y_{l}=\left\{y_{l}, H\right\}_{+}=0,
\end{aligned}
$$

Thus system (2) is realized by constraining $H_{0}$ to $X=\left\{\phi_{1}=\phi_{2}=0\right\}$.

It is also possible to constrain with respect to the standard symplectic structure after a logarithmic change of variables; but then $\phi_{1}$ is no longer a polynomial. 
(ii) Particles on the Circle

Let $\left(a_{1}, \ldots, a_{n} ; b_{1}, \ldots, b_{n}\right) \in\left(R^{+}\right)^{n} \oplus R^{n}$ and let $L$ be the periodic difference operator defined by

$$
L f=\left(a_{l-1} f(l-1)+b_{l} f(l)+a_{l} f(l+1), l \in Z\right),
$$

with $a_{l+n}=a_{l}, b_{l+n}=b_{l}$ and $f(l+n)=f(l)$ for all $l$. We will assume that the spectrum of $L$ is simple except at the very end of the section.

Now let $\lambda_{1}=\lambda_{1}(a ; b), \ldots, \lambda_{n}=\lambda_{n}(a ; b)$ be the eigenvalues of $L$ and $f_{1}, \ldots, f_{n}$ the corresponding eigenfunctions which are determined up to a choice of sign by the normalization $\sum_{i=1}^{n} f_{i}^{2}(l)=1, i=1, \ldots, n$. We have, as before,

$$
b_{l}=\sum_{i=1}^{n} \lambda_{i} f_{i}^{2}(l),
$$

and

$$
a_{l}=\sum_{i=1}^{n} \lambda_{i} f_{i}(l) f_{i}(l+1)
$$

for all $l$. Fix $(\alpha ; \beta) \in\left(R^{+}\right)^{n} \oplus R^{n}$ and set

$$
M=\left\{(a ; b) \in\left(R^{+}\right)^{n} \oplus R^{n}: \lambda_{i}(a ; b)=\lambda_{i}(\alpha ; \beta), \quad i=1, \ldots, n\right\} .
$$

Finally, let $N$ be the quotient of $\left(R^{2} /(0,0)\right)^{n}$ by the fixed point free involutions

$$
\begin{aligned}
& \left(x_{1}, y_{1}, \ldots, x_{i}, y_{i}, \ldots, x_{n}, y_{n}\right) \\
& \longrightarrow\left(x_{1}, y_{1}, \ldots,-x_{i},-y_{i}, \ldots, x_{m}, y_{n}\right),
\end{aligned}
$$

$i=1, \ldots, n$. $N$ is a smooth symplectic manifold since the involutions are symplectic for the usual form $\sum_{i=1}^{n} d x_{i} \wedge d y_{i}$.

Theorem 1. The map

$$
M \ni(a, b) \rightarrow\left(\sqrt{a_{1}} f_{1}(1), \sqrt{a_{1}} f_{1}(2), \ldots, \sqrt{a_{1}} f_{n}(1), \sqrt{a_{1}} f_{n}(2)\right) \in N
$$

is smooth and one to one.

Proof. The map is well defined because $f_{l}(1), f_{l}(2)$ cannot both be zero for any $1 \leqq l \leqq n$. The map is smooth by regular perturbation theory. It is easy to show, by imitating the proof of Theorem 1 for particles on the line ${ }^{14}$, that $(a, b) \in M$ can be constructed from $\left(\sqrt{a_{1}} f_{1}(1), \sqrt{a_{1}} f_{1}(2), \ldots, \sqrt{a_{1}} f_{n}(1), \sqrt{a_{1}} f_{n}(2)\right) \in N$. Of course, this proves that the map is one to one.

$$
f_{l}(1)=\frac{\sqrt{a_{1}} f_{l}(1)}{\sqrt{\sum_{i=1}^{n}\left(\sqrt{a_{1}} f_{i}(1)\right)^{2}}}, \quad f_{l}(2)=\frac{\sqrt{a_{1}} f_{l}(2)}{\sqrt{\sum_{i=1}^{n}\left(\sqrt{a_{1}} f_{i}(1)\right)^{2}}}, \quad l=1, \ldots, n
$$

14 Now the proof begins with the self-evident identities 
Let

$$
x_{l}=\sqrt{a_{1}} f_{l}(1), \quad y_{l}=\sqrt{a_{1}} f_{l}(2),
$$

for $l=1, \ldots, n$. This can only be done locally on $N$.

Theorem. The system of differential equations

$$
\begin{aligned}
& \frac{d}{d t} a_{l}=a_{l}\left(b_{l+1}-b_{l}\right), \\
& \frac{d}{d t} b_{l}=2\left(a_{l}^{2}-a_{l-1}^{2}\right), \quad l=1, \ldots, n,
\end{aligned}
$$

with $a_{0}=a_{n}, b_{n+1}=b_{1}$ is mapped to

$$
\begin{aligned}
& \frac{d}{d t} x_{l}=\left(\frac{\sum_{i=1}^{n} \lambda_{i}\left(x_{i}^{2}+y_{i}^{2}\right)}{\sum_{i=1}^{n} x_{i}^{2}+y_{i}^{2}}-\lambda_{l}\right) x_{l}+\left(\sum_{i=1}^{n} x_{i}^{2}+y_{i}^{2}\right) y_{l}, \\
& \frac{d}{d t} y_{l}=\left(\lambda_{l}-\frac{\sum_{i=1}^{n} \lambda_{i}\left(x_{i}^{2}+y_{i}^{2}\right)}{\sum_{i=1}^{n} x_{i}^{2}+y_{i}^{2}}\right) y_{l}-\left(\sum_{i=1}^{n} x_{i}^{2}+y_{i}^{2}\right) x_{l} .
\end{aligned}
$$

System (2) is invariant under the map

$$
\begin{aligned}
& \left(x_{1}, y_{1}, \ldots, x_{i}, y_{i}, \ldots, x_{n}, y_{n}\right) \\
& \longrightarrow
\end{aligned}\left(x_{1}, y_{1}, \ldots,-x_{i},-y_{i}, \ldots, x_{n}, y_{n}\right),
$$

$1 \leqq i \leqq n$, and therefore defines a flow on $N$.

Proof. The proof is essentially the same as that of Theorem 2 for particles on the line and so we will only sketch it. Again, system (1) can be written in matrix form by setting

$$
L=\left(\begin{array}{ccc}
b_{1} a_{1} & a_{n} \\
a_{1} b_{2} & \ddots & \\
& & b_{n-1} a_{n-1} \\
a_{n} & & a_{n-1} b_{n}
\end{array}\right)
$$

and

$$
B=\left(\begin{array}{rrrr}
0 & a_{1} & & -a_{n} \\
-a_{1} & 0 & \ddots & \\
& & & a_{n-1} \\
a_{n} & & -a_{n-1} & 0
\end{array}\right)
$$


As before $\frac{d}{d t} f_{l}=B f_{l}, 1 \leqq l \leqq n$. It follows that

$$
\begin{array}{ll}
\frac{d}{d t} f_{l}(1)=\left(b_{1}-\lambda_{l}\right) f_{l}(1)+2 a_{1} f_{l}(2), & l=1, \ldots, n \\
\frac{d}{d t} f_{l}(2)=\left(\lambda_{l}-b_{2}\right) f_{l}(2)-2 a_{1} f_{l}(1), &
\end{array}
$$

Now, a direct calculation, using the identities

$$
2 a_{1}=\sum_{i=1}^{n} x_{i}^{2}+y_{i}^{2}
$$

and

$$
\frac{b_{1}+b_{2}}{2}=\frac{\sum_{i=1}^{n} \lambda_{i}\left(x_{i}^{2}+y_{i}^{2}\right)}{\sum_{i=1}^{n} x_{i}^{2}+y_{i}^{2}}
$$

gives (2).

Now let $\phi_{1}=\sum_{i=1}^{n} x_{i} y_{i}, \phi_{2}=\sum_{i=1}^{n} y_{i}^{2}-x_{i}^{2}$ and

$$
H_{0}=-\sum_{i=1}^{n} \lambda_{i} x_{i} y_{i}+\left(\sum_{i=1}^{n} x_{i}^{2}\right)\left(\sum_{i=1}^{n} y_{i}^{2}\right) .
$$

We have

$$
\begin{aligned}
& \left\{\phi_{1}, \phi_{2}\right\}=2 \sum_{i=1}^{n} x_{i}^{2}+y_{i}^{2}, \\
& \left\{\phi_{1}, H_{0}\right\}=0,
\end{aligned}
$$

and

$$
\left\{\phi_{2}, H_{0}\right\}=2 \sum_{i=1}^{n} \lambda_{i}\left(x_{i}^{2}+y_{i}^{2}\right) \text { modulo } \phi_{1}, \phi_{2} .
$$

Also

$$
\left\{x_{l}, H_{0}\right\}=-\lambda_{l} x_{l}+2\left(\sum_{i=1}^{n} x_{i}^{2}\right) y_{l},
$$

and

$$
\left\{y_{l}, H_{0}\right\}=\lambda_{l} y_{l}-2\left(\sum_{i=1}^{n} y_{i}^{2}\right) x_{l},
$$


for $l=1, \ldots, n$. Constraining $H_{0}$ to $X=\left\{\phi_{1}=\phi_{2}=0\right\}$ we find $H=H_{0}+\alpha_{1} \phi_{1}+\alpha_{2} \phi_{3}$ where $\alpha_{1}, \alpha_{2}$ are determined by

$$
\begin{aligned}
& 0=\left\{\phi_{1}, H\right\}=2\left(\sum_{i=1}^{n} x_{i}^{2}+y_{i}^{2}\right) \alpha_{2}, \\
& 0=\left\{\phi_{2}, H\right\}=2 \sum_{i=1}^{n} \lambda_{i}\left(x_{i}^{2}+y_{i}^{2}\right)-2\left(\sum_{i=1}^{n} x_{i}^{2}+y_{i}^{2}\right) \alpha_{1}
\end{aligned}
$$

on $X$ so that $\alpha_{1}=\frac{\sum_{i=1}^{n} \lambda_{i}\left(x_{i}^{2}+y_{i}^{2}\right)}{\sum_{i=1}^{n} x_{i}^{2}+y_{i}^{2}}$ and $\alpha_{2}=0$. Hence the equations of constrained motion are

$$
\begin{aligned}
\frac{d}{d t} x_{l}=\left\{x_{l}, H\right\}= & -\lambda_{l} x_{l}+2\left(\sum_{i=1}^{n} x_{i}^{2}\right) y_{l} \\
& +\left(\frac{\sum_{i=1}^{n} \lambda_{i}\left(x_{i}^{2}+y_{i}^{2}\right)}{\sum_{i=1}^{n} x_{i}^{2}+y_{i}^{2}}\right) x_{l}, \\
\frac{d}{d t} y_{l}=\left\{y_{l}, H\right\}= & \lambda_{l} y_{l}-2\left(\sum_{i=1}^{n} y_{i}^{2}\right) x_{l} \\
& -\left(\frac{\sum_{i=1}^{n} \lambda_{i}\left(x_{i}^{2}+y_{i}^{2}\right)}{\sum_{i=1}^{n} x_{i}^{2}+y_{i}^{2}}\right) y_{l},
\end{aligned}
$$

for $l=1, \ldots, n$. Clearly, the constraints $\phi_{1}, \phi_{2}$ and the free Hamiltonian $H_{0}$ are functions on $N$. Indeed, the entire calculation really takes place on $N$. Thus system (2) is obtained by constraining $H_{0}$ to $X$ inside $N^{15}$.

Once again the constrained motion is integrable.

Theorem 3. The polynomials

$$
E_{k}=x_{k} y_{k}+\sum_{l \neq k} \frac{\left(x_{k} y_{l}-x_{l} y_{k}\right)^{2}}{\lambda_{l}-\lambda_{k}}, \quad 1 \leqq k \leqq n,
$$

Poisson commute on $X$. Moreover, $\left\{H_{0}, E_{k}\right\}_{X}=0, k=1, \ldots, n$. Also

$$
\sum_{k=1}^{n} E_{k}=\phi_{1}
$$

and

$$
\sum_{k=1}^{n} \lambda_{k} E_{k}=\phi_{1}^{2}-H_{0}
$$

15 Note that $2 \sum_{i=1}^{n} x_{i}^{2}=\sum_{i=1}^{n} x_{i}^{2}+y_{i}^{2}=2 \sum_{i=1}^{n} y_{i}^{2}$ 
Proof. The proof is by now a familiar calculation which we omit.

The map of Theorem 1 must be changed when there are double eigenvalues ${ }^{16}$ because of the further ambiguity in the choice of an orthonormal frame of eigenfunctions. It is enough to explain the necessary modifications when there is just one double eigenvalue, say $\lambda \equiv \lambda_{1}=\lambda_{2}$. The choice of an orthonormal pair of eigenfunctions for $\lambda$ is parametrized by SO(2), that is a circle. Now the constraints, free Hamiltonian and motion are rotation invariant. Thus we can remove the ambiguity by taking the quotient of $N$ with respect to the torus action

$$
\begin{aligned}
& \left(x_{1}, x_{2}\right) \rightarrow\left(x_{1} \cos \theta-x_{2} \sin \theta, x_{1} \sin \theta+x_{2} \cos \theta\right), \\
& \left(y_{1}, y_{2}\right) \rightarrow\left(y_{1} \cos \theta-y_{2} \sin \theta, y_{1} \sin \theta+y_{2} \cos \theta\right),
\end{aligned}
$$$$
0 \leqq \theta \leqq 2 \pi
$$

and mapping $M$ into this quotient. We see that one degree of freedom is lost in the particle system for every double eigenvalue.

There is another change of variables under which Toda becomes an integrable system of constrained particles. Let

$$
\sigma_{0}, \sigma_{3}, \sigma_{4}, \sigma_{7}, \sigma_{8}, \ldots ; \quad f_{0}, f_{3}, f_{4}, f_{7}, f_{8}, \ldots ;
$$

and

$$
\sigma_{1}, \sigma_{2}, \sigma_{5}, \sigma_{6}, \ldots ; \quad \mathrm{f}_{1}, \mathrm{f}_{2}, \mathrm{f}_{5}, \mathrm{f}_{6}, \ldots ;
$$

denote the periodic and antiperiodic spectrum and eigenvectors of $L$ respectively. It is well known that $\sigma_{0}>\sigma_{1} \geqq \sigma_{2}>\sigma_{3} \geqq \sigma_{4}>\sigma_{5} \geqq \sigma_{6}>\ldots$. Now, by interpolating the gradient of $\operatorname{det}(L-\lambda)$ off $\sigma_{0}, \sigma_{2}, \sigma_{4}, \ldots$, we obtain the formulae

$$
\begin{aligned}
& \frac{1}{A}=\sum_{i \geqq 0} \varepsilon_{i} f_{2 i}^{2}(l), \\
& \frac{b_{l}}{A}=\sum_{i \geqq 0} \sigma_{2 i} \varepsilon_{i} f_{2 i}^{2}(l)-\frac{2 c}{A}, \\
& \frac{c}{A a_{l}}=\sum_{i \geqq 0} \varepsilon_{i} f_{2 i}(l) f_{2 i}(l+1),
\end{aligned}
$$

where $c, \varepsilon_{1}, \varepsilon_{2}, \ldots$, depend only on $\sigma_{0}, \sigma_{2}, \sigma_{4}, \ldots$, and

$$
A=\prod_{l=1}^{n} a_{l} .
$$

The appropriate change of variables is

$$
(a, b) \rightarrow\left(\sqrt{\varepsilon_{0} A a_{1}} f_{0}(1), \sqrt{\varepsilon_{0} A a_{1}} f_{0}(2), \ldots, \sqrt{\varepsilon_{i} A a_{1}} f_{2 i}(1), \sqrt{\varepsilon_{i} A a_{1}} f_{2 i}(2), \ldots\right)
$$

and Toda is realized by constraining

$$
H_{0}=-\sum_{i \geqq 0} \sigma_{i} x_{i} y_{i}+\left(\sum_{i \geqq 0} x_{i}^{2}\right)\left(\sum_{i \geqq 0} y_{i}^{2}\right)
$$

16 It is clear that $L$ cannot have eigenvalues of multiplicity more than two 
to

$$
\begin{aligned}
\phi_{1} & =\sum_{i \geqq 0} x_{i} y_{i}-c, \\
\phi_{2} & =\sum_{i \geqq 0}\left(y_{i}^{2}-x_{i}^{2}\right) .
\end{aligned}
$$

Finally (see Deift-Trubowitz [1979c]) we note that passing to the continuum limit $\frac{1}{A}=\sum_{i \geqq 0} \varepsilon_{i} f_{2_{i}}^{2}(l)$ converges to the formula $1=\sum_{i \geqq 0} \varepsilon_{i} f_{2_{i}}^{2}(x)$ for the Hills operator [see 9].

\section{References}

1a. Ablowitz, M.J., Kaup, D.J., Newell, A.C., Segur, H.: The inverse scattering transform-Fourieranalysis for nonlinear problems. Std. Appl. Math. 53, 249-315 (1973)

1b. Ablowitz, N.J., Kaup, D.J., Newell, A.C., Segur, H. : Method for solving the Sine-Gordon equation, Phys. Rev. Lett. 30, 1262-1263 (1973)

2. Dirac, P.A.M.: Lectures in quantum mechanics. New York: Yeshiva University 1964

3a. Deift, P., Trubowitz, E. : Inverse scattering on the line. Comm. Pure Appl. Math. 32, 121-251 (1979)

3b. Deift, P., Trubowitz, E.: The phase space geometry for the Korteweg-de Vries equation. To appear

3c. Deift, P., Trubowitz, E.: An identity among squares of eigenfunctions. To appear

4. Kaup, D.J.: Method for solving the Sine-Gordon equation in laboratory coordinates. Stud. Appl. Math. 54, 165-179 (1975)

5. Lax, P.: Periodic solutions of the KdV equation Comm.. Pure Appl. Math. 28, 141-188 (1975)

6. Moser, J.: Finitely many mass points on the line under the influence of an exponential potential. In: Dynamic systems theory and applications, (ed. J.Moser) pp.467-497. New York, Berlin, Heidelberg: 1975

7. Moser, J.: Various aspects of integrable Hamiltonian systems. To appear in Proc. CIME Conference held in Ressanone, Italy, June 1978

8. Neumann, C.: De problemate quodam mechanico, quod ad primam integralium ultraellipticorum classem revocatur. J. Reine Angew. Math. 56, 46-63 (1859)

9. McKean, H.P., Trubowitz, E.: Hill's operator and hyperelliptic function theory in the presence of infinitely many branch points. Comm. Pure Appl. Math. 29, 143-226 (1976)

Communicated by A. Jaffe

Received November 26, 1979 